\title{
An experimental study of wave-in-deck loading and its dependence on the properties of the incident waves
}

\author{
Li Ma ${ }^{\mathrm{a}, *}$ Chris $\mathrm{Swan}^{\mathrm{a}}$ \\ ${ }^{a}$ Imperial College London, South Kensington Campus, London SW7 2AZ, UK
}

\begin{abstract}
Recent advances in the description of extreme ocean waves have led to the definition of more severe design conditions. These changes include increases in the sea-state severity for a given return period, the nonlinear amplification of crest elevations beyond second-order and, perhaps most importantly, the occurrence of wave breaking in both intermediate and deeper waters. These developments raise important questions as to whether present design practice, commonly based upon simplified regular wave theories, provides a realistic estimate of the maximum design loads on fixed offshore structures. This is especially relevant if the applied wave load involves the loss of an effective air-gap and, the occurrence of wave-indeck (WID) loading; the latter believed to be the most common cause of failure in severe seas.

To address these issues, an extensive laboratory study of wID loading has been undertaken. This paper presents the first part of the findings from this study; the aim being to provide an improved physical understanding of WID loading in a wide range of incident wave conditions. The study shows that the applied loads are critically dependent upon both the wave shape and the water particle kinematics arising at the highest elevations within the wave crest; both properties being strongly influenced by the occurrence of wave breaking, particularly wave over-turning. Indeed, the occurrence of wave breaking leads to markedly different load time-histories with important consequences for both the maximum applied load and the onset of a dynamic excitation. The results presented herein provide important guidance as to the effective modelling of these critical loading events.
\end{abstract}

Keywords: wave-in-deck loads, wave impact, extreme wave loads, wave breaking, wave kinematics

\section{Introduction}

The reliability of any offshore structure is dependent upon the applied environmental loads and its structural resistance; the latter required to support the former. In seeking to assess this balance, the primary source of uncertainty lies in the loading. In most cases, particularly in deep and intermediate water depths, the extreme environmental loads are dominated by wave loading. In the case of a fixed structure, this load can be sub-divided into two parts: the sub-structure loads, or the loads acting on that part of the structure which supports the topside, and the wave loads that act directly on the topside. The latter is commonly referred to as wave-in-deck (wID) loading. This component of the total load is the most difficult to predict, associated with the largest uncertainty, and commonly identified as the cause of global structural failure or, at the very least, significant local damage.

Evidence of the importance of WID loading is provided on Figure 1. In both cases the very large WID loads arising due to the inundation of the topside structure, by a large wave crest, were identified as the cause of failure. More broadly, data from the Gulf of Mexico confirms that in total, Hurricanes Katrina \& Rita in 2005 and Gustave in 2008 destroyed 186 platforms and damaged 171 more (U.S. Department 15 of State, 2006; Hall, 2012). In many instances, the failures were directly attributed to WID loading. The challenge posed by WID arises from two key points:

(1) The loads are said to be 'badly behaving'; a very large increase in the applied load arising from a relatively small increase in the level of inundation, $\Delta \eta$. The latter is defined as $\Delta \eta=\eta_{c, \max }-h_{d}$, where $\eta_{c, \max }$ is the maximum incident crest elevation and $h_{d}$ the deck elevation.

\footnotetext{
${ }^{*}$ Corresponding author

Email addresses: li.ma08@imperial.ac.uk (Li Ma), c.swan@imperial.ac.uk (Chris Swan)
} 


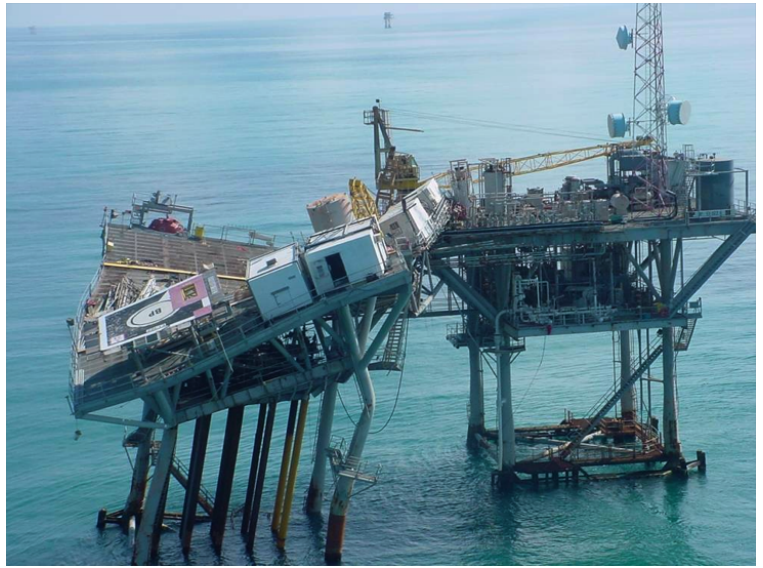

(A)

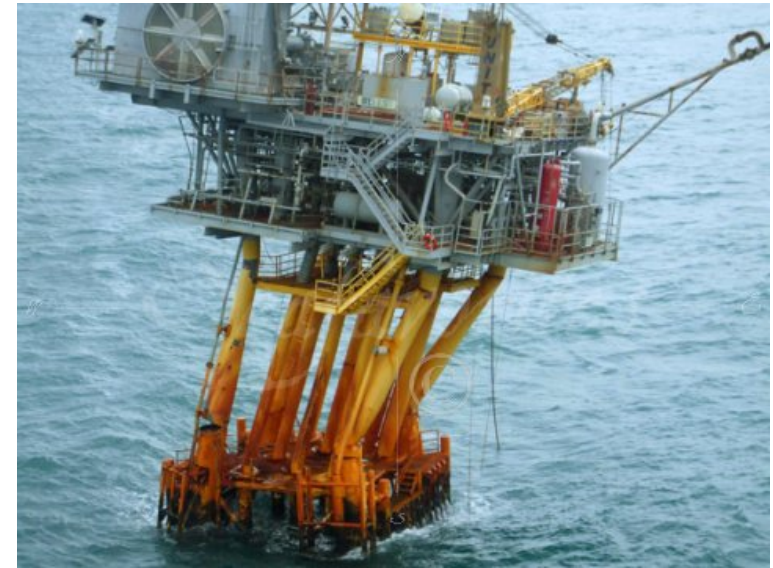

(в)

Figure 1: Examples of damage caused by wID loads in the Gulf of Mexico: (A) two offshore platform damaged during a hurricane off Louisiana (Kaiser and Chambers, 2017) and (в) a platform damaged in Hurricane Ike (Paganie, 2008).

(2) The occurrence of the loads is critically dependent upon the most extreme crest elevations, $\eta_{c, \max }$. By definition, these are subject to the largest statistical and modelling uncertainty.

The combination of (1) and (2) ensures that the magnitude of any WID loads will be difficult to predict and potentially very large. As a result, offshore structures are not typically designed to withstand these loads, but rather to avoid them. This is achieved by setting a deck elevation, $h_{d}$, such that an effective underside of the deck structure. When this is achieved, wID loading ceases to be a problem, removing an important cause of failure. Whilst this can be achieved for a new-build structure, it may not be relevant for an existing structure that requires re-appraisal, particularly if this is part of a planned life extension. In such cases, the deck elevation may be insufficient to avoid WID loads. This can arise for 30 several reasons:

(a) With the sea-state severity defined in terms of a spectral peak period $\left(T_{p}\right)$ and a significant waveheight $\left(H_{s}\right)$, recent research suggests an increase in the crest elevations for a given exceedance probability. Evidence to support this comes from both laboratory (Latheef and Swan, 2013) and field (Christou and Ewans, 2014) data.

(b) For a given return period, there has been a small but progressive increase in the severity of the predicted sea states (defined by $H_{s}$ ). This has largely arisien due to improvementns in long-term predictions; see, for example, Young et al. (2011) and Tychsen et al. (2016).

(c) Regulatory change has involved the increasing adoption of a $10^{-4}$ rather than a $10^{-2}$ annual exceedance probability.

40 (d) In some instances sea bed settlement, arising from the de-pressurisation of the underlying reservoir, has led to reduced deck elevations.

If one or more of these factors is relevant, the effective air-gap will be reduced and an assessment of WID loading becomes essential. The purpose of the present paper is to describe a new laboratory study designed to explore the fundamental characteristics of wiD loads. Specifically, it will address the 45 variation in the loads with the crest elevation and hence the level of wave inundation, the wave shape, the near-surface water particle kinematics, the directional spread and the occurrence of wave breaking. These are key features of the extreme waves that are likely to initiate wID loading. Importantly, they are also features that differ markedly from the regular waves that form the basis of most simplified WID load models presently used in design practice. As such, the insights provided are directly relevant to the 


\section{Background}

Graaf et al. (1995) were the first to describe WID in terms of a transfer of momentum from the wave to the topside structure; the applied force, $\mathbf{F}(t)$, being equal to the rate of change of momentum, $\frac{\partial \mathbf{J}}{\partial t}$, such that

$$
\mathbf{F}(t)=\frac{\partial \mathbf{J}}{\partial t}=\frac{\partial}{\partial t}(m \mathbf{u})
$$

where $m$ is the mass and $\mathbf{u}$ the velocity vector $\mathbf{u}=(u, v, w)$. Taking the $x$-component of the force gives

$$
F_{x}(t)=\frac{\partial}{\partial t}(m u)=u \frac{\partial m}{\partial t}+m \frac{\partial u}{\partial t} \approx u \frac{\partial m}{\partial t}=u \cdot \rho \mathbf{u A},
$$

where $\frac{\partial u}{\partial t} \rightarrow 0$ high in the wave crest; hence the a quasi-stationary assumption adopted by Graaf et al. (1995). Within Equation (2), $\frac{\partial m}{\partial t}$ is the mass flow rate with components $\left(\rho u A_{x}, \rho w A_{z}\right)$, where $A_{x}$ is the area on the front face and $A_{z}$ the area on the underside of the desk structure, through which the fluid enters. Adopting this approach it is clear that the applied horizontal force, $F_{x}$, is dependent upon both the water particle kinematics, $\mathbf{u}$, and the shape of the incident wave crest, $\eta(x, y, t)$; the relative importance of these terms being one of the issues that needs to be addressed.

Other predictive WID load models include Kaplan et al. (1995) and the silhouette method; the latter incorporated in the API (2014), ISO (2013) and NORSOK (2017) international standards and the DNV-GL (2019b) recommended practice. In comparing these solutions, the Kaplan et al. (1995) model 65 expresses the horizontal load as the sum of an impact and drag load, while the silhouette method defines the maximum load as a heavily calibrated drag term; further details of both solutions given in DNV-GL (2019b). Whilst both the Kaplan et al. (1995) and the silhouette methods are used in engineering practice, the Graaf et al. (1995) model consistently addresses the total momentum-flux, providing important physical insights appropriate to the analysis of the present data.

wID loading will, by definition, be associated with the largest waves in a severe sea-state. As such, the incident wave will lie in the tail of the relevant crest-height distribution. Earlier work (Latheef and Swan, 2013) has shown that such waves will experience the competing effects of nonlinear amplification and wave breaking. In terms of the non-linearity, this arises at $2^{\text {nd }}$-order and above; the extent of the higherorder effects depending on the directional spread. In intermediate and deep water, this non-linearity 75 involves a shift of energy to the higher frequencies. Since these terms are predominantly in-phase with the freely-propagating wave components, they produce higher and steeper crests, which are spatially more compact, evolve more rapidly, and incorporate larger near-surface water particle kinematics. These characteristics will all affect the magnitude of the applied wiD loads.

Furthermore, increases in both the wave steepness and the water particle kinematics will drive the process of wave breaking. This also has important implications for wID loads. Whilst wave breaking is expected in shallower water coastal locations, recent studies (Latheef and Swan, 2013; Latheef, 2014) have

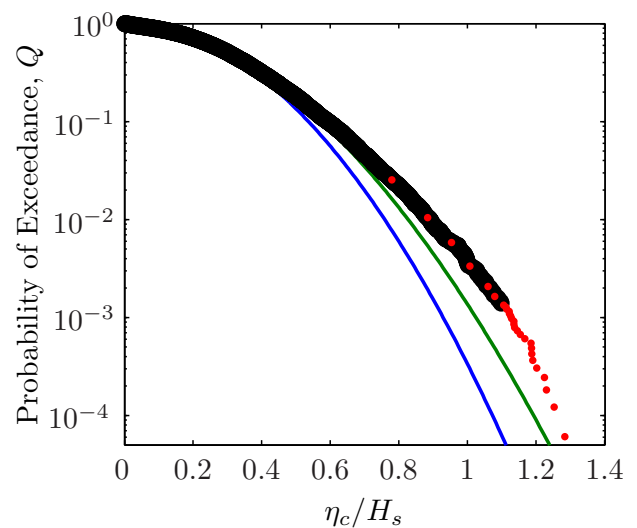

(A) $H_{s}=0.15 \mathrm{~m}$

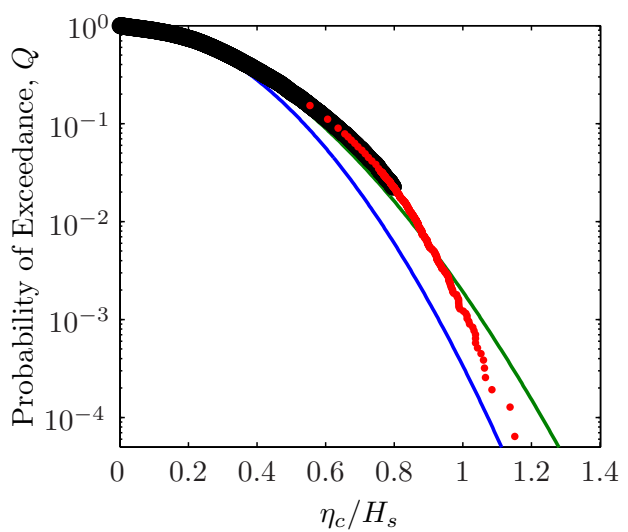

(в) $H_{s}=0.20 \mathrm{~m}$.

Figure 2: Normalised crest-height distribution for uni-directional JONSWAP sea-states with $T_{p}=1.6 \mathrm{~s}$ Latheef (2014). Experimental data $\left([\bullet]\right.$ non-breaking and $[\bullet]$ breaking events) compared to the equivalent predictions from $\left[\boldsymbol{L}^{-}\right]$the Rayleigh and [ $]$ second-order distributions. 
shown that it will also affect the largest crest-heights in intermediate and deep water depths (Figure 2). This raises important questions concerning the validity of present WID load models which are commonly based on regular or steady wave solutions. Since such solutions are not able to accurately describe the

85 wave shape, the water particle kinematics or the occurrence of wave breaking, it is unclear how they can be expected to give accurate load predictions without extensive and problem-specific calibration. The present paper will address this point, by considering the variation in the WID loads for a broad range of incident wave forms.

The paper continues with details of the laboratory study $(\S 3)$ and preliminary observations $(\S 4)$. The 90 main results are presented in $\S 5$ and $\S 6$. These are followed by some concluding remarks which seek to emphasis the practical implication of the data presented.

\section{Experimental apparatus and test conditions}

\subsection{Laboratory wave basin}

The present study was undertaken in the directional wave basin located in the Hydrodynamics Laboratory within the Department of Civil and Environmental Engineering at Imperial College London. This facility (Figure 3) has a plan area of $20 \mathrm{~m} \times 10 \mathrm{~m}$, with the (adjustable) water depth being set to $1.5 \mathrm{~m}$. It is equipped with 56 dry-backed, flap-type wave paddles; each paddle $0.35 \mathrm{~m}$ in width and bottom hinged $0.7 \mathrm{~m}$ below the still water level (SWL). A perforated parabolic beach is installed on the opposite side to the wave-makers. This dissipates the wave energy, preventing unwanted reflections. The side-walls of the basin are constructed from glass for maximum optical access. As shown in Figure 3, the global coordinate system is defined such that the $x$-axis is aligned with the mean direction of wave propagation (perpendicular to the front face of the paddles), the $y$-axis defines the transverse direction, and the $z$-axis (not shown) points vertically upward.

The wave paddles are digitally controlled and accurately generate waves within the frequency range $0.3 \mathrm{~Hz} \leqslant f \leqslant 3 \mathrm{~Hz}$, with angles of propagation of up to $\pm 45^{\circ}$ relative to the $x$-axis. A force feed-back loop forms an integral part of the control system, enabling the active absorption of any (small) reflected wave energy. While the beach is most effective at absorbing high-frequency wave components, the active absorption capabilities of the paddles work best for lower frequencies. Combining the two absorption mechanisms, previous studies by Masterton and Swan (2008) confirmed that the reflection is typically maintained below $5 \%$ and does not build up with time. Further details regarding the success of the forcecontrolled wave generation and absorption system are fully documented by the studies of Spinneken and Swan (2009a,b, 2012).

\subsection{Wave measurement}

The incident wave conditions were first measured in the absence of a model topside (see below) using an array of 49 resistance-type wave gauges; the array covering the plan area of the model topside. The gauges formed a $7 \times 7$ grid with a uniform spacing of $\Delta x=\Delta y=100 \mathrm{~mm}$. Each gauge was made from two pre-tensioned $0.5 \mathrm{~mm}$-diameter steel wires, spaced $15 \mathrm{~mm}$ apart. These allow the surface elevation to be recorded with an accuracy of $\pm 0.5 \mathrm{~mm}$ when sampled at $128 \mathrm{~Hz}$. The small diameter of the wires caused little or no disturbance of the free surface, while the pre-tensioning removes any dynamic excitation of the wave gauges with steep (possibly breaking) incident waves. With the incident wave forms fully defined, the wave gauge array was removed and its space re-occupied by the model topside. The incident wave cases were then re-generated and the corresponding WID loads measured. In undertaking this process, a $50^{\text {th }}$ wave gauge, placed outside the plan area of the topside, remained in place to serve as an assurance of the repeatability of the incident wave conditions. Full verification of the wave generation and the repeatability of the measurements is provided in Masterton and Swan (2008), Latheef and Swan (2013) and Ma (2017).

\subsection{Load measurement}

The WID load measurement system consisted of three main parts: a height-adjustable supporting frame to enable the variation of the deck elevation $\left(h_{d}\right)$, a 6-degree-of-freedom (6-DOF) load cell and a modular topside structure. The model structure and the associated instrumentation were installed at the centre of the wave basin, connected to a rigid gantry spanning over the basin. An overall view of the installation within the wave basin is provided in Figure 3, while a close-up is given in Figure 4(A). 
Figure 4(в) offers a detailed view of the 6-DOF load cell providing the time-histories of the global WID loads. This unit was designed specifically for the present study. In total, it utilises eight Kistler ${ }^{T M}$ piezo-electric force sensors, four rotational bearings and eight linear bearings. The connection details of each sensor were designed to avoid interference or 'mechanical cross-talk' between the load components. These consist of three force components $\left(F_{x}, F_{y}, F_{z}\right)$ and three moment components $\left(M_{x}, M_{y}, M_{z}\right)$. Together, the sensors allow the simultaneous measurements of all six load components with a uniform sample rate of $12 \mathrm{k} \mathrm{Hz}$ on all channels. This high sample rate is essential for the measurement of wid loads, particularly in breaking waves; the latter likely to produce loads that are impulsive and therefore rapidly changing. Each load cell was supplied pre-calibrated. This was checked using a static system calibration showing excellent agreement and linearity within the measurement range. Whilst the complete details of the system are provided in Ma (2017), the success of the design is clearly demonstrated in Table 1; the application of a load in any one DOF having a negligible response in all other loading components.

\subsection{Model topside}

The model topside was rigidly connected to the base of the load cell (Figure 4(A)); the connection being made through a pair of plates that allow the topside to be rotated relative to the mean incident wave direction $(x)$. The model was constructed using a grid of perforated aluminium panels (Figure 4(A),(C)). This design allows the topside porosity to be varied in an easily quantifiable manner; achieved by either changing the internal spacing of the panels, or utilising panels of different surface porosity. For the tests considered in the present paper, all panels had a constant $63 \%$ open area; the panels spaced $50 \mathrm{~mm}$ apart

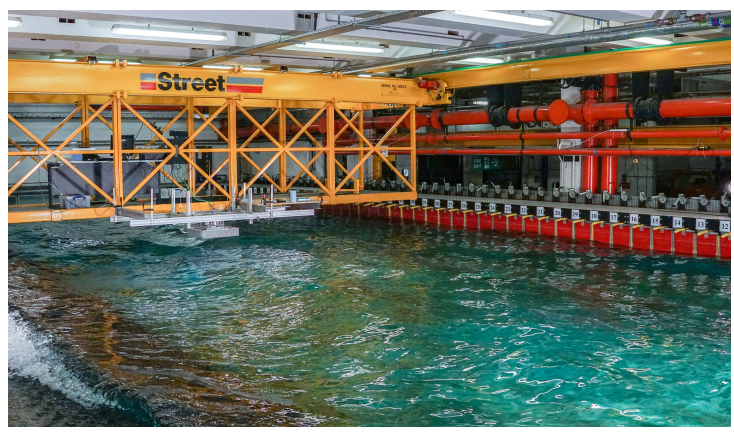

(A) general set-up.

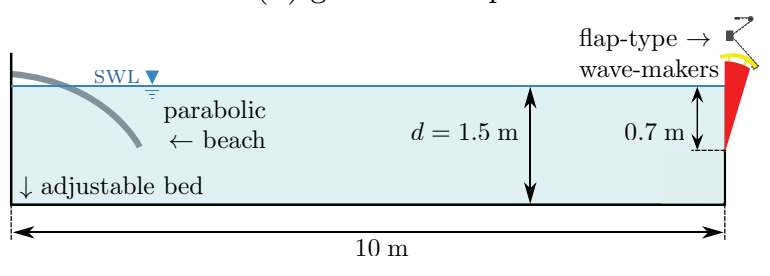

(B) side elevation.

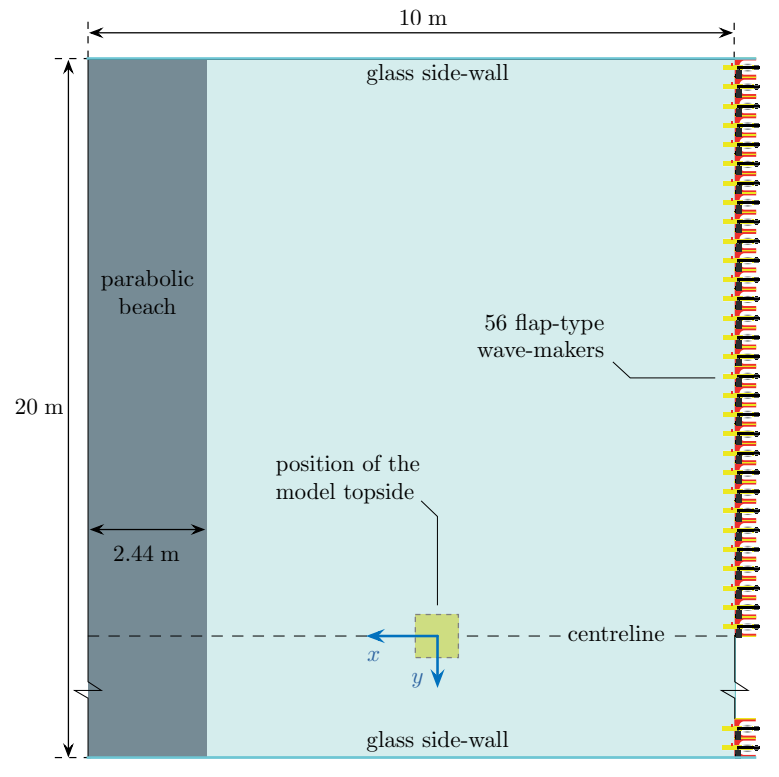

(c) plan view.

Figure 3: Photo and schematics of the wave basin at Imperial College London.

\begin{tabular}{c|c|cccccc}
\hline & \multicolumn{7}{c}{ Application } \\
\cline { 3 - 8 } & & $F_{x}\left[N^{-1}\right]$ & $F_{y}\left[N^{-1}\right]$ & $F_{z}\left[N^{-1}\right]$ & $M_{x}\left[(N m)^{-1}\right]$ & $M_{y}\left[(N m)^{-1}\right]$ & $M_{z}\left[(N m)^{-1}\right]$ \\
\hline \multirow{5}{*}{ Response } & $F_{x}[N]$ & $\mathbf{0 . 9 9 8 6}$ & 0.0026 & 0.0062 & 0.0046 & 0.0028 & 0.0002 \\
& $F_{y}[N]$ & 0.0062 & $\mathbf{0 . 9 9 8 2}$ & 0.0022 & 0.0055 & 0.0043 & 0.0224 \\
& $F_{z}[N]$ & 0.0049 & 0.0032 & $\mathbf{1 . 0 0 7 7}$ & 0.0087 & 0.0017 & 0.0116 \\
& $M_{x}[N m]$ & 0.0000 & 0.0006 & 0.0001 & $\mathbf{0 . 9 9 9 6}$ & 0.0046 & 0.0018 \\
& $M_{y}[N m]$ & 0.0001 & 0.0001 & 0.0007 & 0.0002 & $\mathbf{1 . 0 0 0 1}$ & 0.0013 \\
& $M_{z[N m]}[$ & 0.0004 & 0.0007 & 0.0002 & 0.0003 & 0.0012 & $\mathbf{0 . 9 9 9 0}$ \\
\hline
\end{tabular}

Table 1: Typical responses when a loading component is applied to the measurement system. 


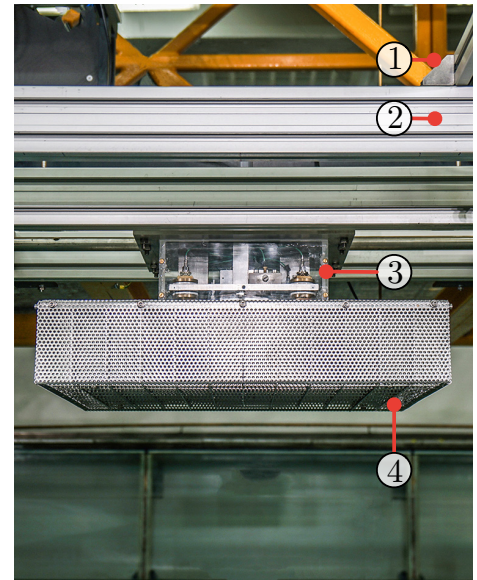

(A) complete system.

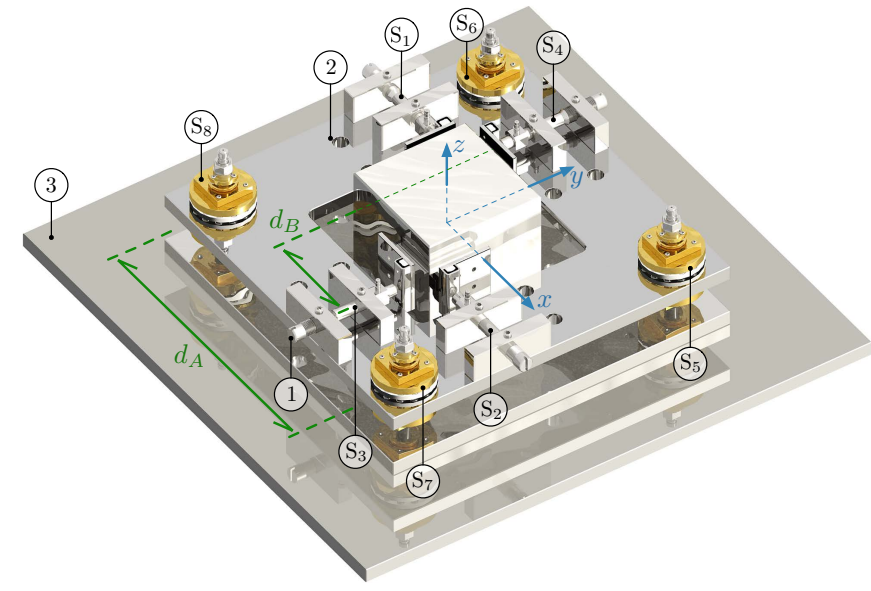

(в) 6-DOF load cell.

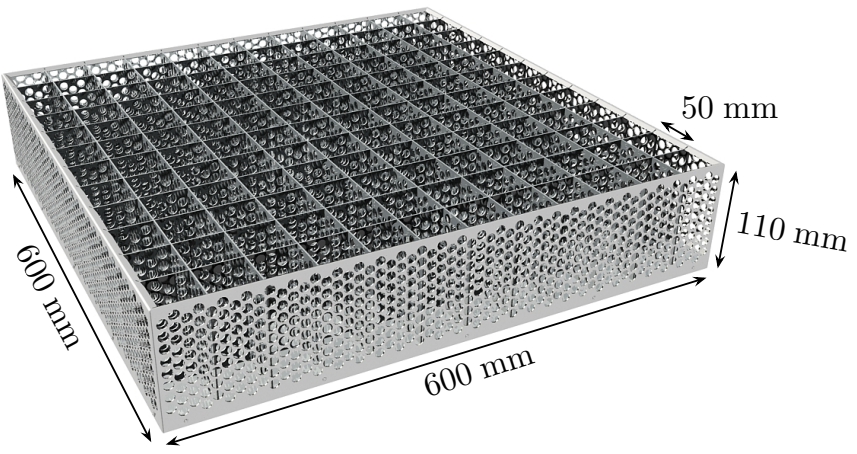

(c) schematic of the modular topside.

Figure 4: The WID load measurement system. (A) The complete system installed within the wave basin: (1) rigid support (gantry), (2) height-adjustable supporting frame, (3) 6-DOF load cell and (4) modular topside. (B) Details of the 6-DOF load cell: $\left(S_{1-8}\right)$ the individual force sensors, (1) sensor connection, $(2)$ connection to the external frame and (3) rotation plate. (C) The assembled modular model topside noted as (4) in (A).

in both horizontal directions. This choice of open area (or porosity) was adopted to be representative of a relatively open topside structure; typical varying from $40 \%-80 \%$, unless they are fully/partially plated. The model topside had a plan area of $600 \times 600 \mathrm{~mm}$. With an adopted length-scale of $l_{s}=1: 100$ (see below), this corresponds to $60 \times 60 \mathrm{~m}$ which is again representative of realistic deck structures.

The majority of the test cases included within this study relate to a deck elevation of $h_{d}=125 \mathrm{~mm}$ (or $12.5 \mathrm{~m}$ at full-scale). This was chosen to be a representative lower-bound, particularly for structures that have suffered some level of sea bed subsidence and are therefore most vulnerable to WID loading. In adopting this lower-bound value, the broadest possible range of incident wave conditions, capable of producing WID loads, could be investigated.

\subsection{Digital imagery}

High-speed image sequences were captured using a SONY NEX-FS700R high-definition video recorder. The free-field wave profiles were recorded at 240 FPS through the glass (basin) side wall. These images provide important confirmation of the wave gauge data, especially for the over-turning wave cases where measurement uncertainties increase. In contrast, the WID loading events were recorded at 480 FPS. These images were recorded in addition to the global WID load measurements; the purpose being to document any local flow effects, particularly the occurrence and extent of any wave breaking.

\subsection{Test conditions}

Given the objectives of the present study, focused (deterministic) wave events were adopted throughout. These provide the best-controlled experimental environments; the isolated wave events allowing a systematic variation in the wave properties. The focused waves were all based upon JonswAP spectra 
following Hasselmann et al. (1973). With the amplitude of the individual frequency components proportional to the spectral shape, $S_{\eta \eta}(\omega)$, these deterministic events are representative of the shapes of the largest wave events arising in a random sea-state following Lindgren (1970), Boccotti (1983) and 175 Tromans et al. (1991). In addition, some regular wave cases were included; the local wave periods and crest elevations being based upon a number of focused wave cases, facilitating direct comparisons. A summary of the incident wave conditions (and deck elevations) is provided in Table 2. By adopting a length-scale of $l_{s}=1: 100$ and maintaining Froude number similarity (giving a time-scale of $t_{s}=1: 10$ ), the generated test cases represent a broad range of practically relevant wave conditions. Taken together, the parameter ranges indicated on Table 2 define more than 100 test cases. These include both nonbreaking and different types of breaking waves; the latter including limiting, spilling and over-turning waves.

In generating the individual wave events, the incident wave spectra were discretised within a frequency range of $26 / 64 \mathrm{~Hz} \leqslant f \leqslant 3 / T_{p}$. Adopting a frequency resolution of $\Delta f=1 / 64 \mathrm{~Hz}$, each wave case was described by at least 81 frequency components. The location of the desired incident wave crest was then adjusted, iteratively, to coincide with the front face of the model topside. This was achieved by uniformly and linearly offsetting the starting phase of each individual frequency component. In generating the video records noted in $\S 3.5$, the incident wave events were also shifted laterally (in $y$ ) to occur at the side wall.

\subsection{Nonlinear wave simulations}

For each focused wave case generated in the present study, a corresponding fully-nonlinear numerical simulation was also performed to define the underlying water-particle kinematics. A Multiple-flux Boundary Element Method (BEM) was adopted for the simulations. This assumes the flow is irrotational and solves the classical Green's second identity on the boundaries of the physical domain. Full details of the method are provided in Hague and Swan (2009) (2D), Christou (2009) and Archibald (2011) (3D). Evidence of the success of this method is given in Figure 5. This is taken from Haley (2016), concerns a very steep breaking wave event and contrasts the BEM solution with data describing both the surface elevation, $\eta(t)$, and the water particle kinematics; the latter based upon LASER-Doppler Anemometry (LDA) measurements.

\section{Preliminary data}

This section presents an analysis of preliminary data appropriate to the quality/accuracy of the laboratory study. Its focus lies in the measured WID loading; the success of the wave generation having already been established in earlier work by Masterton and Swan (2008), Latheef and Swan (2013) and Ma (2017).

\subsection{Load time-histories, $F(t)$}

An ideal load measuring system would have infinite stiffness. Since this is not practically achievable, some system response is inevitable; the extent of the response depending on both the dynamic properties of the measurement system and the nature of the applied loads. If $F_{I}(t)$ is the applied (or input) load and $\mathcal{H}(t)$ the system unit-impulse response function, the recorded load, $F_{R}(t)$, is the time-domain convolution of $F_{I}(t)$ and $\mathcal{H}(t)$. When expressed in the frequency domain, $f$, this relationship is defined as:

$$
F_{R}(f)=F_{I}(f) \cdot \mathcal{H}(f) .
$$

\begin{tabular}{r|c}
\hline level of inundation & $10 \mathrm{~mm} \leqslant \Delta \eta \leqslant 50 \mathrm{~mm}$ (where $\left.\Delta \eta=\eta_{c, \text { max }}-h_{d}\right)$ \\
deck elevation & $95 \mathrm{~mm} \leqslant h_{d} \leqslant 145 \mathrm{~mm}$ from swL \\
spectral peak period & $T_{p}=1.4 \mathrm{~s}, 1.6 \mathrm{~s}$ and $1.8 \mathrm{~s}$ \\
directional spread & $0^{\circ} \leqslant \sigma_{\theta} \leqslant 30^{\circ \dagger}$ and Ewans (1998) spreading \\
spectral bandwidth & $\gamma=1,2.5,5$ and regular waves \\
\hline
\end{tabular}

Table 2: The range of parameters (in model-scale) considered in the present laboratory study. $\dagger$ Frequency-independent Gaussian spreading with zero-mean and a standard deviation of $\sigma_{\theta}$. 


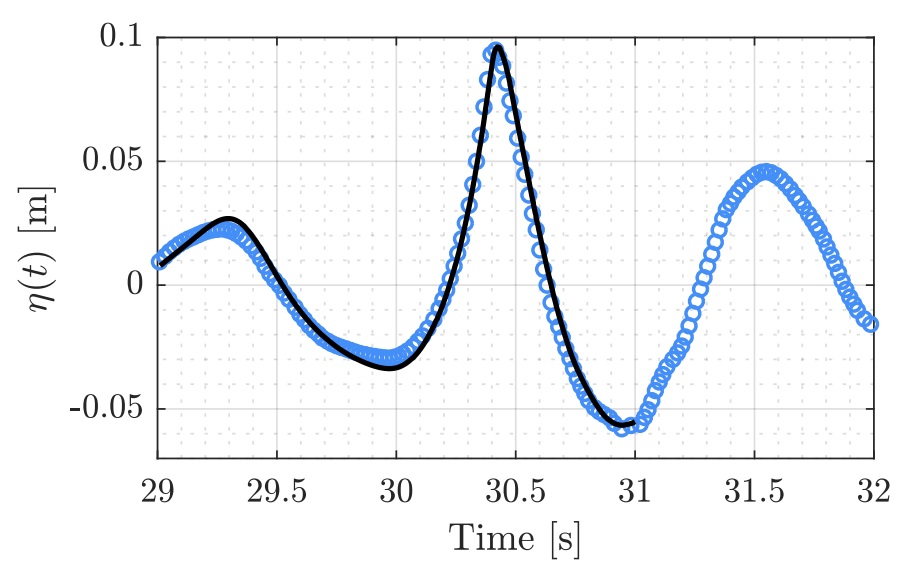

(A)

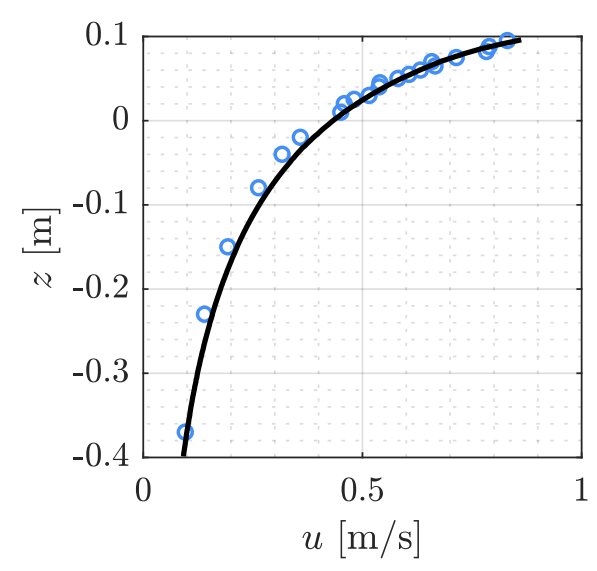

(B)

Figure 5: Comparisons between [o] experimental measurements of a breaking wave and simulations for both (A) the surface elevation and (B) the water particle kinematics under the largest wave crest (Haley, 2016).

In practice, the required information is $F_{I}(t)$, but the recorded information is $F_{R}(t)$; the difference between the two being due to the system response. In many cases, this difference is minimised by ensuring that the natural frequency $\left(f_{n}\right)$ of the measurement system lies significantly above the highest frequency of the applied load. However, since the present study includes the possibility of impulsive or slamming loads, this is not possible. As such, the measured data needs to be 'filtered' to remove the response of the measurement system. This was achieved using two approaches. The first models each DOF independently as a linear mass-spring-damper system, commonly known as a 'structural filter'. In this case the system transfer response function, $\mathcal{H}(f)$, can be approximated by:

$$
\mathcal{H}(f)=f_{n}^{2} /\left[\left(f_{n}^{2}-f^{2}\right)+2 \zeta f f_{n} i\right]
$$

where $\zeta$ is the damping ratio. The expression was developed by Haley et al. (2014) based on Clough and Penzien (2003). Using this, Equation (3) can be re-arranged to define the applied load, $F_{I}(t)$; the calculations being performed in the frequency domain.

The second approach was based upon a time-domain moving-average filter. The span of the filter was chosen as the damped natural period, $T_{d}=1 / f_{d}$, where $f_{d}$ is the damped natural frequency. As a result, the filter performs best around frequencies related to the response of the system. To ensure that the impulsive nature of the applied loads was unaffected, the filter was only applied after the initial onset of the loading event. The start-time for this filter was defined by an up-crossing in which the recorded force exceeds $0.5 \mathrm{~N}$ immediately before reaching the maximum; the entire loading event being subject to the moving-average.

In order to apply the above methods, the damping ratio $(\zeta)$ was defined in terms of the logarithmic decrement, $\delta$; the latter calculated from the response time-history under free vibration, having applied an initial impulse. The relevant equations are given by:

$$
\zeta=\frac{\delta}{\sqrt{4 \pi^{2}+\delta^{2}}}, \quad \text { where } \quad \delta=\frac{1}{n} \ln \left|\frac{F_{i}}{F_{n+i}}\right|,
$$

and $F_{i}$ and $F_{n+i}$ are the maximum loads after $i$ and $(n+i)$ oscillations, respectively. In addition, the undamped natural frequency was defined by:

$$
f_{n}=f_{d} / \sqrt{1-\zeta^{2}}
$$

where $f_{d}$ was derived from a Fast Fourier Transform (FFT) of the impulse-response time-history and was defined as the frequency corresponding to the largest amplitude.

The success of these alternative filtering approaches is addressed in Figures 6 and 7 . Figure 6 presents the response spectra of three repeated 'hammer tests'; the latter involving the application of an impact load using a small-sized, rubber-ended hammer. The data relates to the primary loading component, 


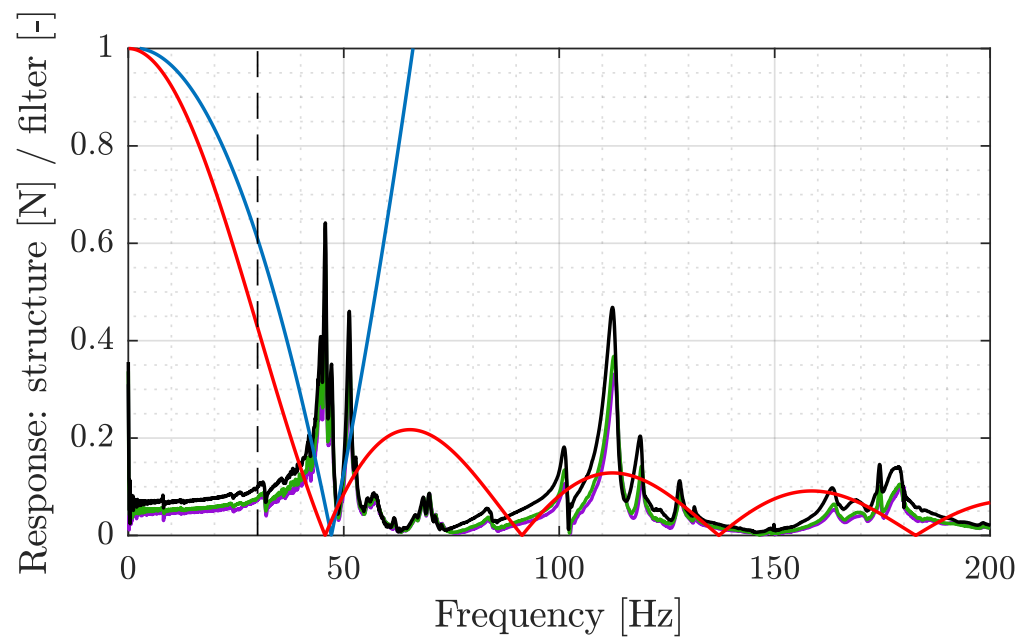

Figure 6: The response spectra of [- - — - - ] three repeated hammer tests and comparisons to the frequency responses of alternative filters: [- $]$ a single-Dof structural filter and [ - ] a moving average filter. [ -- ] The highest loading frequency that is of interest.

$F_{x}$, and is derived from a Fourier analysis of the resulting load time-history. Several important points can be observed. First, the hammer tests are highly repeatable. Second, for the model topside under consideration, $f_{d}$ is approximately $48 \mathrm{~Hz}$. Third, comparisons with the frequency responses of the two filters show that the primary dynamic responses will be effectively removed. Moreover, for $f<30 \mathrm{~Hz}$ the data lie outside the frequency range where the input signal is significantly attenuated. This frequency is highlighted on Figure 6 and relates to the highest loading frequency; the cause being the passage of the wave front through successive panels. Interestingly, such loads only arise in non-breaking wave events, where the applied loads are non-impulsive and, consequently, there is very little dynamic excitation of the load measurement system to be removed.

Comparisons between the outputs from the two filters are given in Figure 7. Sub-plot (A) relates to a non-impulsive load caused by a non-breaking wave event and sub-plot (В) an impulsive load arising from a large breaking wave. Both cases relate to $F_{x}(t)$ since this is the most impulsive loading component and, consequently, the most difficult to analyse. In effect, this represents a lower-bound to the filter performance. In the non-impulsive load case (Figure 7(A)) the system exhibits little excitation and the results arising from the two filters are in close agreement. However, as the loads become impulsive, the performance of the structural filter diminishes (Figure $7($ в)). The explanation for this is that $\mathcal{H}(f)$ attenuates frequencies below $f_{n}$, but amplifies frequencies above. Furthermore, as the applied loads

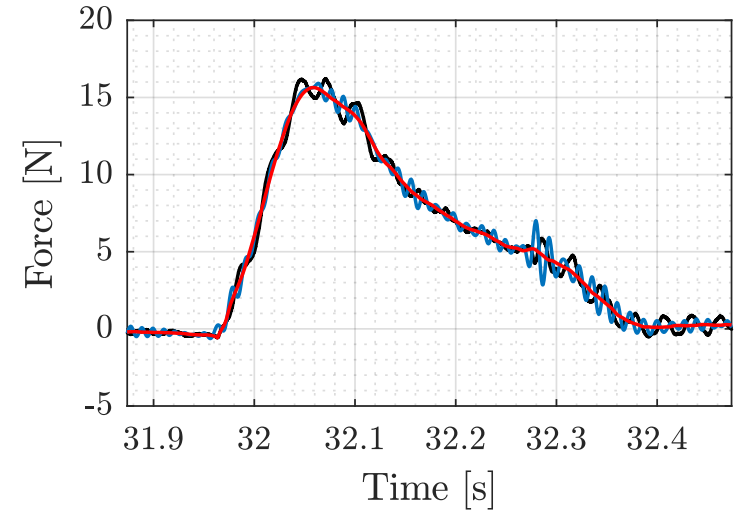

(A)

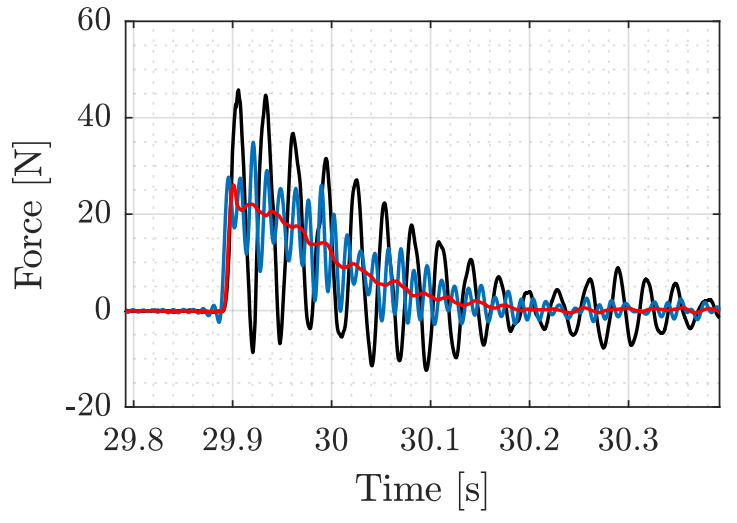

(B)

Figure 7: Comparisons of the two filters: $[-]$ the un-processed load time-history, $[-]$ the load time-history after applying a structural filter, and [- ] the load time-history processed using a moving-average filter. (A) relates to a typical non-impulsive load case, whereas (B) involves a typical impulsive load case. 


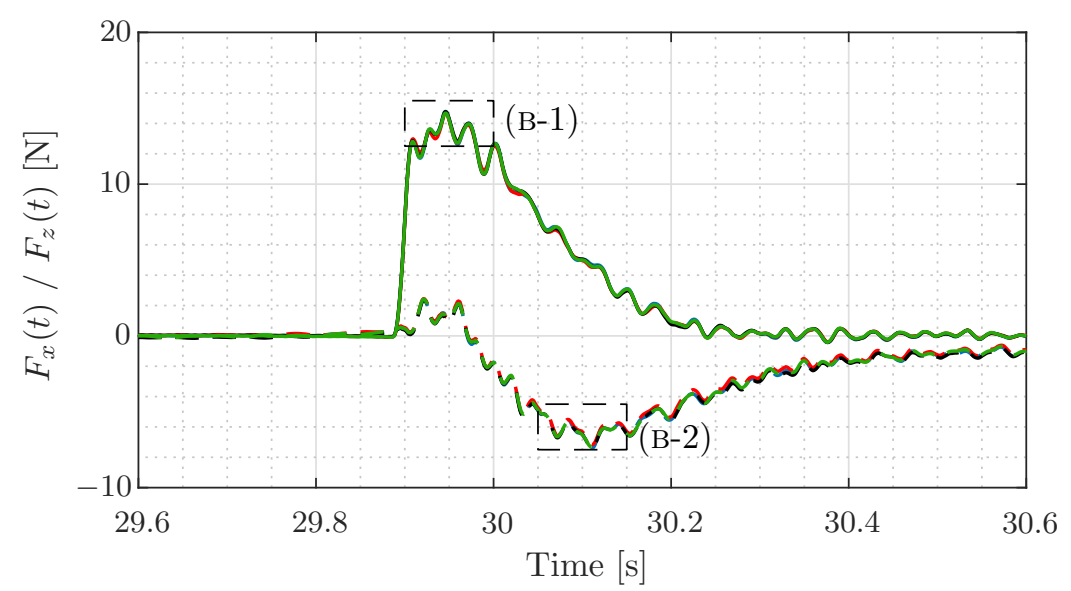

(A)
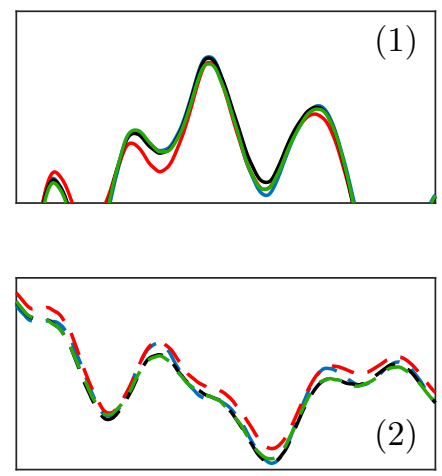

(в)

Figure 8: (A) Four repeated runs of an impulsive load case, demonstrating the repeatability of load measurements of [ $[-]$ $F_{x}$ and $[--] F_{z}$. (B) close-ups of (A).

become large and impulsive, the supporting framework and the gantry are (inevitably) excited. Due to the stiffness of these supporting structures, more high-frequency components are added and these will be amplified. Most importantly, the entire system, now expanded to include the supporting structures, ceases to behave as the sum of several linear single-DOF systems. As a result, Equation (4) no longer applies. In contrast, the second (moving-average) filter performs well for all loading cases (Figure 7). Given its ease of use and wide applicability, this method was consistently adopted throughout the present study. Furthermore, the fact that the two methods are shown to be in close agreement when it comes to the initial rise in the applied load suggests that the choice of the start-time is not significant.

\subsection{Repeatability}

To systematically explore the variations in the WID loads with the incident wave properties, deterministic focused waves are adopted. If this approach is to be successful, the measured load time-histories must be highly repeatable. Evidence of this is given in Figure 8. This provides repeated measurements of $F_{x}(t)$ and $F_{z}(t)$ for an impulsive load case arising in a breaking wave. This represents one of the most difficult load cases to measure and analyse. Despite the fact that these four repeated measurements were taken weeks apart, and based upon different calibrations of the load measurement system, they show close agreement.

\section{WID loads in non-breaking waves}

\subsection{A typical load time-history}

In discussing the present results, attention will be focused on the horizontal component of the WID loads in the mean wave direction, $F_{x}$. This is both the largest component of the applied load and the component most associated with failure. Figure 9 provides a sequence of still images describing a typical WID loading event in which the maximum incident crest elevation, $\eta_{c, \max }$, occurs on the front face of the structure. The corresponding load record, $F_{x}(t)$, is given in Figure 10 and can be characterised by three stages:

(1) Wave entry. This stage covers the interval when the wave enters the topside structure through the front face. It begins when the water surface elevation first exceeds the elevation of the underside of the deck $\left(\eta>h_{d}\right)$ and ends when $\eta$ drops back below $h_{d}$ at the front face. During this interval water enters the topside through both the front face and the underside. At the beginning of this stage, both the mass flow rate and the maximum underlying water particle velocities ('kinematics') are increasing. As a result, this stage is characterised by a relatively sharp rise of the load from zero to $F_{x, \max }$, followed by a slower decrease as the wave crest passes the front face. 


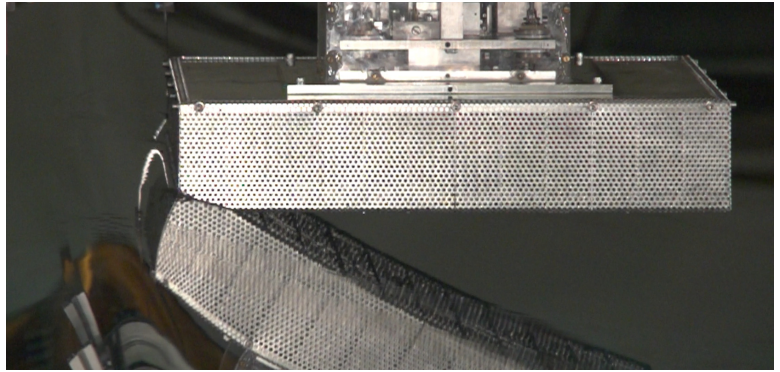

(A)

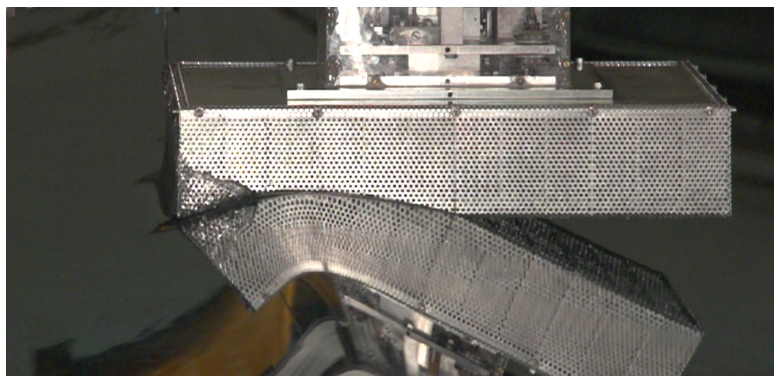

(c)

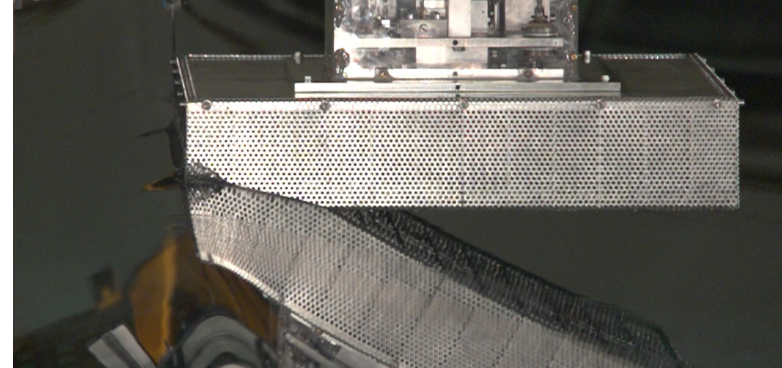

(B)

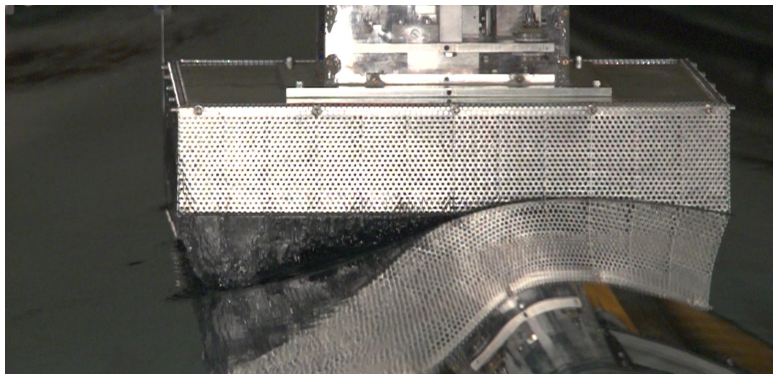

(D)

Figure 9: A sequence of still images describing a typical non-breaking WID loading event, with (A) showing a short moment after the wave has entered the model topside, (B) the maximum crest elevation, $\eta_{c, \text { max }}$, at the front face of the structure, producing the maximum applied load, $F_{x, \max }$, (C) the wave profiling leaving the front face and (D) the wave profile reaching the back face and starting to exit the structure.

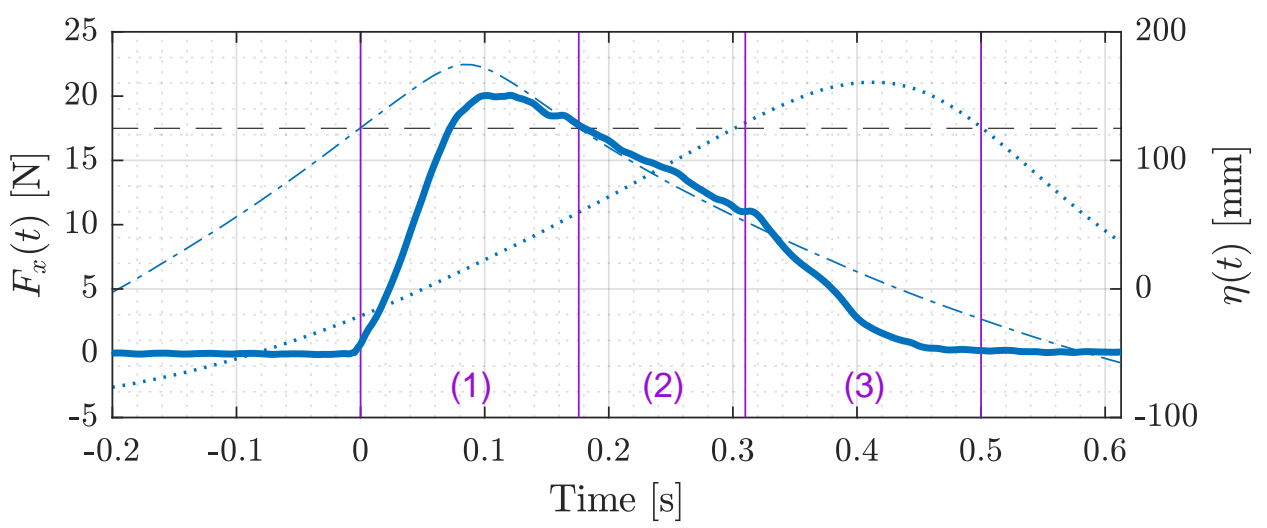

Figure 10: The three stages of the loading process described in relation to the incident wave profile, [ $F_{x}(t),[-\cdots] \eta(t)$ at the front face of the topside, $[\cdots] \eta(t)$ at the back face of the topside, [ --$]$ the deck underside elevation, $h_{d}$.

(2) Wave propagation within the deck. This stage corresponds to the wave profile leaving the front face, propagating through the deck, and reaching the back face of the topside. During this stage, additional water only enters the deck through the underside. As the wave evolves, both the water particle kinematics and the mass flow rate reduce, leading to a gradual reduction in $F_{x}$.

(3) Wave exit. Following immediately after Stage (2), this stage ends with the wave profile exiting the topside. During this stage, little water enters the topside structure. As a result, $F_{x}$ quickly reduces back to zero and completes the loading cycle.

The three stages outlined above establish the general characteristics of the loading process. When 
combined with the description of the WID loads given in Equation (2), the close relationship between the incident wave properties and the resulting $F_{x}(t)$ is clearly established. In adopting this relationship, it should be noted that whilst $\Delta \eta$ is the most commonly adopted indicator of the incident wave shape, and hence the effective $\mathbf{A}$, it only relates to the inundation on the front face. As such, other descriptions of the incident wave shape (or size) may be required, due to the fact that water does not solely enter the topside through the front face. Nevertheless, it is clear that the key quantities determining the rate of change of momentum (and hence the applied loads) concern both the wave shape/size and the water particle kinematics.

\subsection{Level of inundation}

A direct relationship between $F_{x, \text { max }}$ and $\Delta \eta$ is commonly adopted in design practice; the convenience of this approach being the ability to relate the applied loads to the distribution of extreme crest-heights. The relationship between $F_{x}(t)$ and $\Delta \eta$ is considered in Figure 11(A); the increasing $\Delta \eta$ achieved by gradually increasing $\eta_{c, \max }$, while keeping $h_{d}$ and all other parameters constant. As expected, wID loads of increasing magnitude are produced. This is a direct result of both the increased mass flow rate and the larger near-crest kinematics. In addition, it should be noted that for smaller incident waves, loading Stages (2) and (3) are less distinguishable in terms of $F_{x}(t)$. This is due to the wave profile exiting the topside before (or just after) reaching the back face.

The increasing magnitude of the WID loading is also shown in Figure 11(B), with $F_{x \text {, max }}$ plotted as a function of $\Delta \eta$. To assess the relative importance of the wave shape and the kinematics, some normalisation (or de-trending) of the measured loads was performed and is shown alongside the measured data. For example, in taking the normalisation with respect to $\Delta \eta$ (indicated by * $0^{*}$ ), the $\Delta \eta$ for each load case was expressed as a fraction of the largest $\Delta \eta$, and the measured $F_{x, \max }$ divided by the corresponding fraction. This defines one possible $\widehat{F}_{x, \max }$ which is normalised, but not non-dimensionalised. In this case, it removes the linear dependence of $F_{x, \max }$ on $\Delta \eta$ such that if the former were linearly dependent upon the latter, $\widehat{F}_{x, \max }$ would be horizontal. In the present case (Figure 11(B)), this is not the case, suggesting that $\Delta \eta$ alone does not explain the variation in $F_{x \text {,max }}$. A similar normalisation of the data with respect to the square of the maximum horizontal water particle velocity, $u_{\max }^{2}$, is indicated by ... This shows even less deviation from the original measured $F_{x \text {,max }}$, suggesting that the influence of kinematics is perhaps less important compared to that of the incident wave shape. However, when $F_{x, \text { max }}$ is normalised by the product of $\Delta \eta$ and $u_{\max }^{2}$, as indicated by $-\bullet-, \widehat{F}_{x \text {, max }}$ is near horizontal, confirming the importance of the combined influence of these quantities. This, in turn, confirms the dependence of the WID loads, specifically $F_{x, \max }$, on the momentum-flux as outlined in Equation (2).

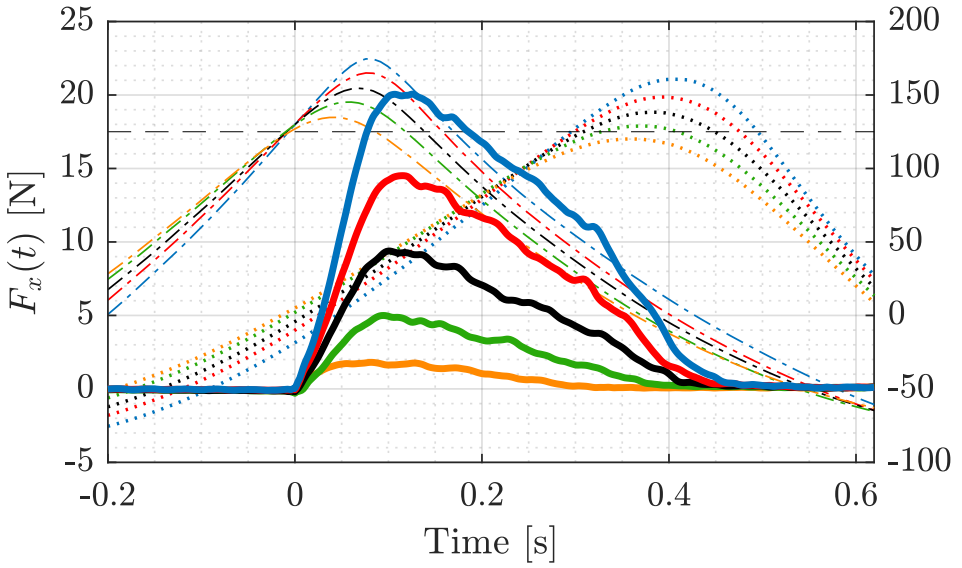

(A)

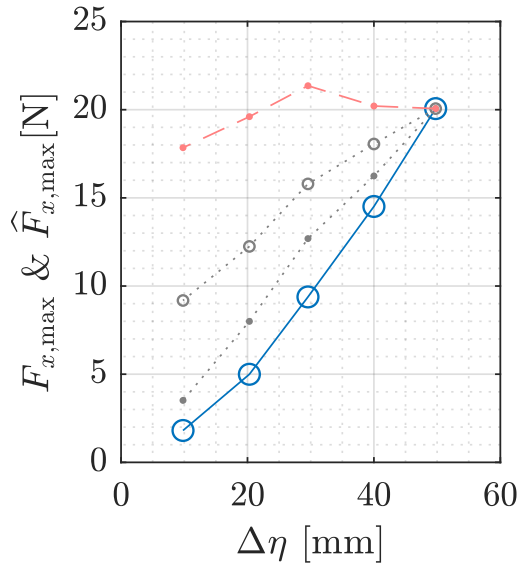

(B)

Figure 11: Horizontal wiD loads, $F_{x}$, for increasing incident crest elevations, $\eta_{c, \text { max }}$, while adopting a constant $h_{d}=125$ mm $[--]$ and JonswAP spectra with $\gamma=2.5, T_{p}=1.6 \mathrm{~s}$ and $\sigma_{\theta}=0^{\circ}$. (A) [ -$] F_{x}(t),[-\cdot-] \eta(t)$ at the front and $[\cdots]$ the back faces of the topside. The colours refer to different $\eta_{c, \text { max }}: 135 \mathrm{~mm}, 145 \mathrm{~mm}, 155 \mathrm{~mm}, 165 \mathrm{~mm}$ and $175 \mathrm{~mm}$. (в) [ - ] The measured $F_{x, \text { max }}$ compared to the normalised forces, $\widehat{F}_{x, \text { max }}$; the normalisation performed with respect to $\left[\cdots \circ \cdot \Delta \eta,[\cdots \cdot] u_{\max }^{2}\right.$ and $[-\bullet-]\left(\Delta \eta \times u_{\max }^{2}\right)$. 


\subsection{Spectral peak period}

In long random wave simulations, the same $\Delta \eta$ does not necessarily cause the same $F_{x, \max }$, but produces a scatter around a mean $F_{x, \max }$. Part of the explanation for this lies in the fact that although $\Delta \eta$ is constant, the underlying kinematics and other aspects of the wave shape can be quite different. In terms of deterministic focused wave events, an example of this is to adopt different spectral peak periods $\left(T_{p}\right)$, while keeping all other wave parameters constant. As a result, the waves vary in steepness while maintaining a constant $\Delta \eta$. Three wave cases corresponding to $T_{p}=1.4 \mathrm{~s}, T_{p}=1.6 \mathrm{~s}$ and $T_{p}=1.8 \mathrm{~s}$ are presented in Figure 12(A), together with the measured horizontal loads. Although the loads are of a similar magnitude, a systematic decrease in $F_{x, \max }$ with increasing steepness (smaller $T_{p}$ ) is clearly observable. To explain these differences, it is necessary to understand that as the incident wave steepness increases, two important changes occur:

(1) The near-surface water particle kinematics increases, and

(2) The width of the wave crest in the direction of wave propagation $\left(\lambda_{c, x}\right)$ reduces.

While (1) increases the rate of momentum transfer, and hence the applied loads, (2) does the opposite. Given these competing effects, the results shown in Figure 12(A) appear to indicate that the increase in the kinematics is unable to compensate for the change in the wave shape. To investigate this further, Figure 12 (в) compares the measured $F_{x, \max }$ with some normalised values, $\widehat{F}_{x, \max }$. With $\Delta \eta$ held constant, the maximum volume of water within the topside $(\Delta \mathcal{V})$ was adopted as a measure of the wave shape. Interestingly, when $F_{x, \max }$ is normalised by $\Delta \mathcal{V}$, as explained previously, the relative magnitudes of $F_{x, \max }$ for the three load cases are reversed (Figure 12(в)). This confirms the critical influence of the incident wave shape. Moreover, a further normalisation of the data based upon $\Delta \mathcal{V} \times u_{\max }^{2}$ is also given in Figure 12(в). The fact that, in this case, the magnitudes of the normalised loads now become almost constant, confirms that the remaining differences in the applied loads are indeed due to the underlying kinematics. Nevertheless, it is also relevant to note that the shape of the loading time-history will remain dependent on the wave steepness, particularly in the $T_{p}=1.4 \mathrm{~s}$ case. This will undoubtedly be important when considering any dynamic excitation.

\subsection{Directional spread}

In addition to the above noted changes, the incident wave form can also be varied in the transverse direction by changing the directional spread. This effect is considered in Figure 13(A). Taken together, the incident waves relate to increasing levels of directional spread, $\sigma_{\theta}$; the latter applied uniformly to all frequency components. All other parameters, including $\Delta \eta$, are held constant. The resulting load

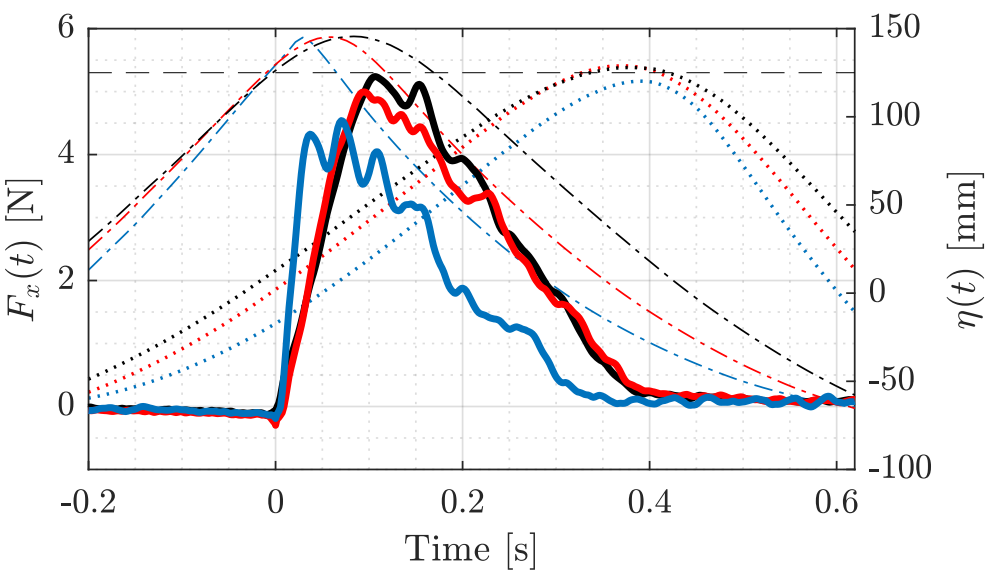

(A)

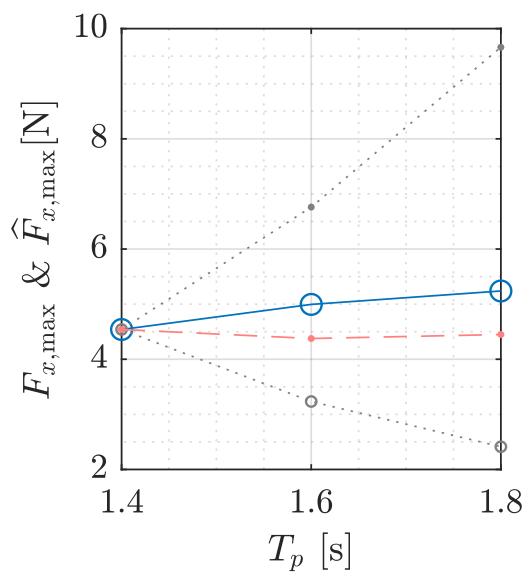

(B)

Figure 12: Horizontal WID loads, $F_{x}$, for varying spectral peak periods, $T_{p}$, with a constant $\Delta \eta\left(\eta_{c, \max }=145 \mathrm{~mm}\right)$, JONSWAP spectra with $\gamma=2.5$ and $\sigma_{\theta}=0^{\circ}$. (A) $\left[-\frac{-}{-}\right] h_{d}=125 \mathrm{~mm},\left[\stackrel{-}{-} F_{x}(t),[-\cdot-] \eta(t)\right.$ at the front and [ $\left.\cdots\right]$ the back faces of the topside. The colours refer to different $T_{p}: 1.4 \mathrm{~s}, 1.6 \mathrm{~s}$ and $1.8 \mathrm{~s}$. (B) [ - ] The measured $F_{x, \max }$ vs. $T_{p}$, compared to the normalised forces, $\widehat{F}_{x, \max }$; the normalisation performed against $\left[\cdots \bullet^{\cdot}\right] \Delta \mathcal{V},\left[\cdots \bullet \cdot u_{\max }^{2}\right.$ and $[-\bullet-]$ $\left(\Delta \mathcal{V} \times u_{\max }^{2}\right)$. 
time-histories show similar characteristics. However, $F_{x, \max }$ gradually reduces as the incident waves incorporate more directional spread. This trend is more clearly observed in Figure $13(\mathrm{~B})$; the $\sigma_{\theta}=20^{\circ}$ case falling slightly out of line because of a marginally lower $\eta_{c \text {,max }}$ compared to the other cases. In considering these load cases, it is again relevant to note the actual changes in the incident wave as $\sigma_{\theta}$ increases:

(1) The transverse crest-length $\left(\lambda_{c, y}\right)$ reduces.

(2) The wave steepness reduces leading to an increase in the width of the wave crest in the direction of wave propagation, $\lambda_{c, x}$. This is clearly shown in Figure 13(A).

(3) With the reduced wave steepness, the near-surface water particle kinematics are also reduced.

Comparing the maximum wetted area on both the front face and on the underside of the topside structure indicates that Points (1) and (2) act counter to each other. As a result, the overall influence of the variations in wave shape is limited. This is confirmed in Figure 13(B), where the decreasing trend of $F_{x, \max }$ with $\sigma_{\theta}$ clearly remains after being normalised by $\Delta \mathcal{V}$. In contrast, the effect of reducing kinematics appears to be responsible for the reduced loading. Evidence to support this view is again produced by the normalised data presented on Figure 13(B).

\subsection{Spectral bandwidth}

Figure 14(A) concerns changes in the applied WID loads arising due to variations in the bandwidth of the underlying JONSWAP spectrum. This is achieved by modifying the peak enhancement factor, $\gamma$; with all other parameters held constant. In making these comparisons, a regular wave case is included. This represents the narrowest-possible bandwidth, with only one freely-propagating frequency component; the period of the latter being based upon the local up-crossing period corresponding to $\eta_{c, \max }$ from the $\gamma=2.5$ case. The load time-histories corresponding to these wave cases are given in Figure 14(A); a comparison between the various cases showing a systematic trend in which the larger the spectral bandwidth, the smaller the applied load. In attempting to provide a physical explanation for this, both the wave shape and kinematics again need to be considered. With a larger spectral bandwidth:

(1) The wave evolves more rapidly. In particular, in the $\gamma=1$ case, the wave crest exits the topside before reaching the back face. As a result, $F_{x}(t)$ is less sustained, exhibiting both a sharper rise and a faster decay.

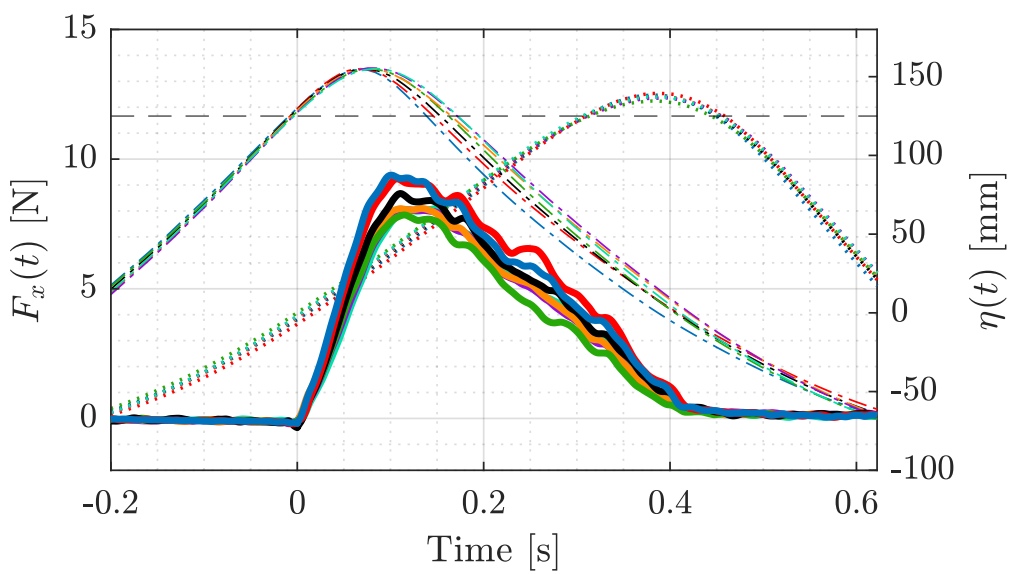

(A)

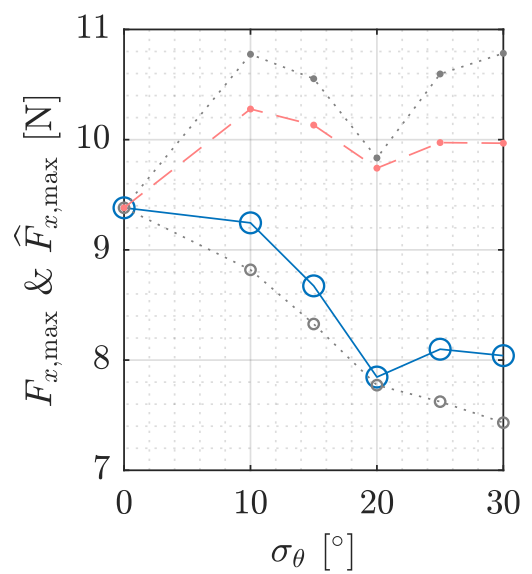

(B)

Figure 13: Horizontal WID loads, $F_{x}$, for increasing levels of directional spread, $\sigma_{\theta}$, with a constant $\Delta \eta, \eta_{c, \max }=155 \mathrm{~mm}$ and JONSWAP spectra with $\gamma=2.5$ and $T_{p}=1.6 \mathrm{~s}$. (A) $[--] h_{d}=125 \mathrm{~mm},[\square] F_{x}(t),[-\cdot-] \eta(t)$ at the centre of the front and $[\cdots]$ ] the back faces of the topside. The colours refer to different $\sigma_{\theta}: 0^{\circ}, 10^{\circ}, 15^{\circ}, 20^{\circ}, 25^{\circ}$ and $30^{\circ}$. (в) $\left[-\_\right]$The measured $F_{x, \max }$ vs. $\sigma_{\theta}$, compared to the normalised forces, $\widehat{F}_{x, \text { max }}$; the normalisation performed with respect to $\left[\cdots 0^{\cdot}\right] \Delta \mathcal{V},\left[\cdots \cdot u_{\max }^{2}\right.$ and $\left[\bullet^{-}\right]\left(\Delta \mathcal{V} \times u_{\max }^{2}\right)$. 


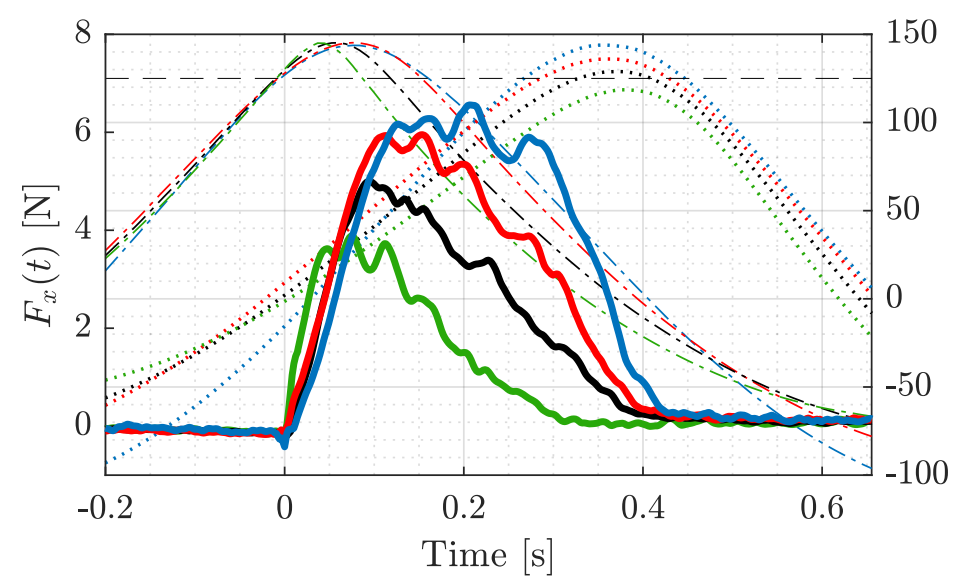

(A)

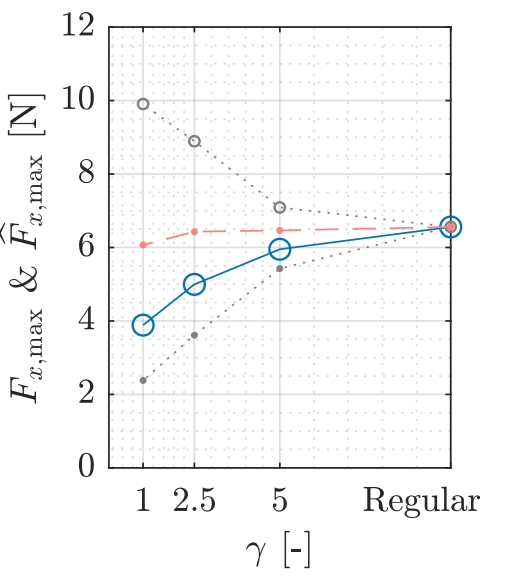

(B)

Figure 14: Horizontal WID loads, $F_{x}$, for a varying spectral bandwidth, $\gamma$, with a constant $\Delta \eta, \eta_{c, \text { max }}=145 \mathrm{~mm}$ and JONSWAP spectra with $T_{p}=1.6 \mathrm{~s}$ and $\sigma_{\theta}=0^{\circ}$. (A) $[--] h_{d}=125 \mathrm{~mm},[-] F_{x}(t)$, [ - - - ] $\eta(t)$ at the front and $[\cdots]$ the back faces of the topside. The colours refer to different spectral bandwidth: regular wave, $\gamma=5, \gamma=2.5$ and $\gamma=1$. (в) [- $]$ The measured $F_{x, \max }$ vs. spectral bandwidth, compared to the normalised forces, $\widehat{F}_{x, \text { max }}$; the

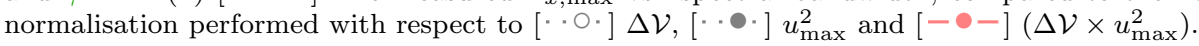

(2) The wave profile is steeper high in the wave crest. This is due to the increased energy content in the higher frequencies and the increased nonlinear interactions between these components. This results in a smaller $\lambda_{c, x}$ and hence a 'thinner' incident wave crest.

(3) The water particle kinematics high in the wave crest will be larger due to the increased importance of the high-frequency components.

The data presented in Figure 14 again emphasise the competing influence of changes in the wave shape and the water particle kinematics. With increases in the spectral bandwidth, the former produces a smaller rate of momentum transfer and hence smaller loads, while the latter produces an increase in both. In comparing these changes, the wave shape exerts the greater influence. Evidence of this is provided by the fact that the regular wave case is associated with the smallest underlying kinematics, but produces the largest $F_{x, \max }$. This point is further confirmed in Figure 14(B) which presents both the maximum measured load, $F_{x, \max }$, as a function of $\gamma$ and the relevant normalisations, $\widehat{F}_{x, \max }$, in respect of both $\Delta \mathcal{V}, u_{\max }^{2}$ and $\left(\Delta \mathcal{V} \times u_{\max }^{2}\right)$. These results show that $F_{x, \max }$ is under the combined influence of the wave shape and the associated kinematics, but that the former is more important.

In summarising the non-breaking wave cases considered thus far, it is clear that having explored a wide range of very distinct wave properties, the WID loads arising can be expressed (explained) in terms of the change in two variables: the incident wave shape and the near-surface water particle kinematics. These affect the rate of the momentum transfer between the fluid and the structure and hence the applied loads. Whilst the water particle kinematics are undoubtedly important, the incident wave shape exerts a dominant or controlling influence on the applied loads. Furthermore, although the level of inundation $(\Delta \eta)$ is important, the wave shape/size in both the in-line $(x)$ and the transvers $(y)$ directions also plays a significant role.

\section{The additional effects of wave breaking}

In practice, an incident wave crest that is sufficiently high to cause wID loads is also very likely to be breaking, or on the point of breaking. This has been discussed earlier; strong evidence being provided in Figure 2 that the low-probability end of a representative crest-height distribution is dominated by breaking waves. As such, the effects of wave breaking should not be overlooked in modelling WID loads. This section revisits some of the issues discussed above; the purpose being to demonstrate the significant influence that wave breaking has on the measured wiD loads.

In order to aid these discussions, several widely considered wave cases are listed in Table 3 . They all arise in JONSWAP spectra with $\gamma=2.5$ and cover a range of breaking wave types; a photograph of each 
type being given in Figure 15. In considering these events, a 'limiting' wave refers to a case where the wave is on the point of breaking or (in some instances) shows the initial onset of small-scale spilling. To highlight the differences caused by wave breaking, Figure 16 provides a sequence of still images depicting a WID loading event caused by an over-turning wave event. Comparisons between these images and those given on Figure 9 highlight the local changes in the wave shape. The extent to which this changes the WID loading time-history, $F_{x}(t)$, is discussed as follows.

\subsection{Level of inundation}

Figure $17(\mathrm{~A})$ presents a set of six load cases in which $\Delta \eta$ is the only variable, making it directly comparable to Figure 11(A). However, the associated $T_{p}$ has been reduced from $1.6 \mathrm{~s}$ to $1.4 \mathrm{~s}$. As a result, the incident wave events are steeper and wave breaking progressively occurs as $\eta_{c, \max }$ increases. The cases considered cover a full range of breaking wave types. In comparing the load time-histories, $F_{x}(t)$, one obvious difference lies in the initial rise of the applied loads (Stage 1), particularly in the overturning wave cases. When wave breaking occurs, the corresponding load records begin with a markedly larger gradient. In effect, the loads become more impulsive as the wave breaking strengthens. Indeed, in the over-turning wave cases, the initial increases in the applied loads are near-vertical. In practice, this characteristic has significant implications for the dynamic excitation of a structure; the latter being very important when it comes to ultimate structural failure.

Further evidence of the importance of wave breaking is provided on Figure 17(B). This directly concerns $F_{x, \max }$ and, as expected, it again shows a marked increase with $\Delta \eta$. To provide a complete comparison, the equivalent data for $T_{p}=1.6 \mathrm{~s}$ and $T_{p}=1.8 \mathrm{~s}$ are also included; the increase in $T_{p}$ causing a reduction in steepness and, consequently, the absence of wave breaking. Interestingly, $F_{x, \text { max }}$ agrees quite well across the three data-sets $\left(T_{p}=1.4 \mathrm{~s}, 1.6 \mathrm{~s}\right.$ and $\left.1.8 \mathrm{~s}\right)$, until the point at which significant wave over-turning occurs. At this point, $F_{x, \max }$ starts to increase much more rapidly with $\Delta \eta$. Indeed, for the over-turning wave cases, the loads are, on average, $30 \%$ larger than non-breaking waves of the same

\begin{tabular}{c|ccc}
\hline Case №. & $T_{p}[\mathrm{~s}]$ & $\eta_{c, \max }[\mathrm{mm}]$ & breaking type \\
\hline C03 & 1.4 & 145 & limiting \\
C04 & 1.4 & 155 & spilling \\
C05 & 1.4 & 156 & over-turning \\
C32 & 1.6 & 155 & non-breaking \\
C44 & 1.8 & 155 & non-breaking \\
\hline
\end{tabular}

Table 3: A list of several widely-considered wave cases; all relating to uni-directional JONSWAP spectra with $\gamma=2.5$.
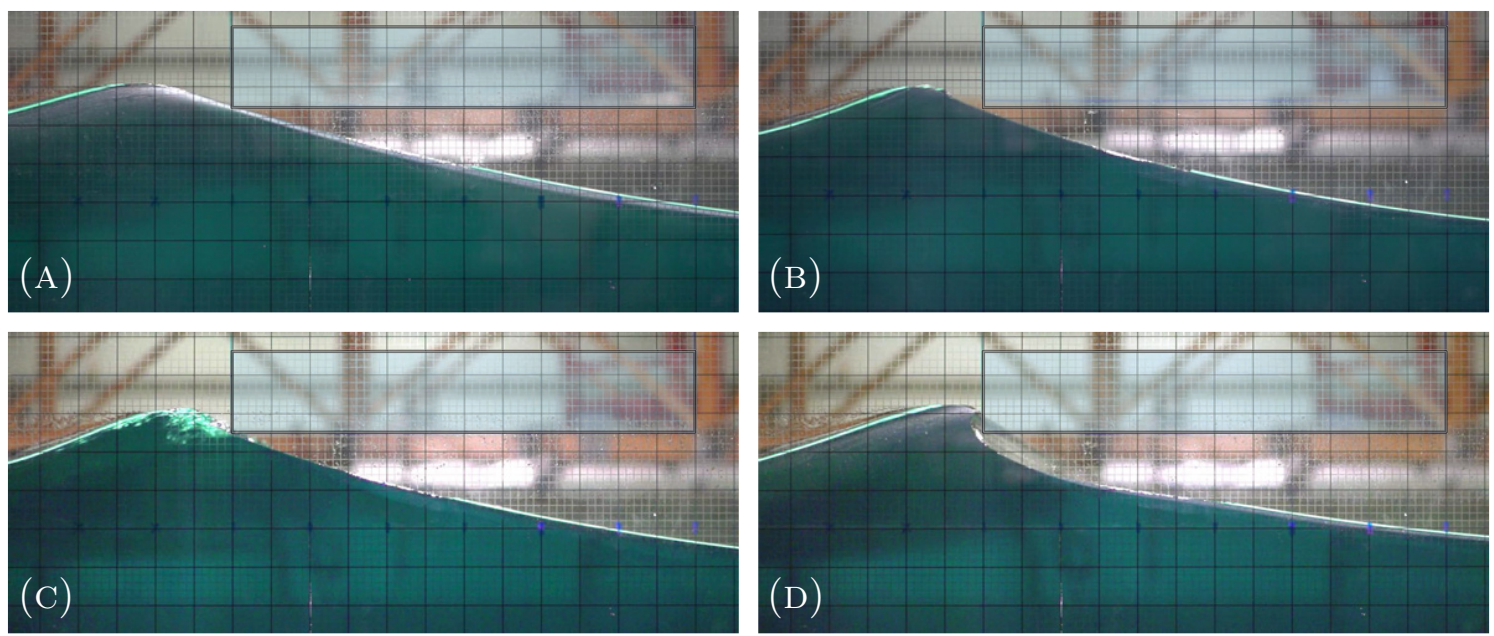

Figure 15: Photographs of the wave shape as it approaches the topside, showing different levels of wave breaking: (A) non-breaking (C32), (B) limiting (C03), (C) spilling (C04), (D) over-turning (C05). In each case the shaded box represents the topside structure. 


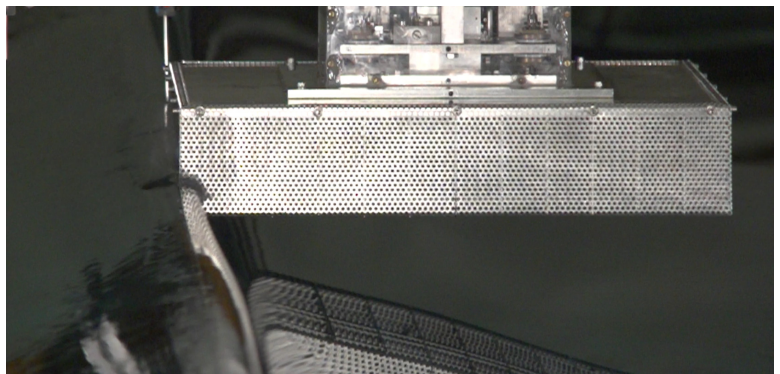

(A)

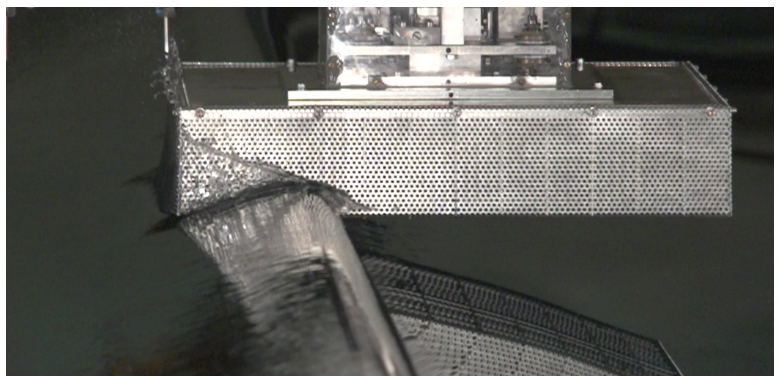

(c)

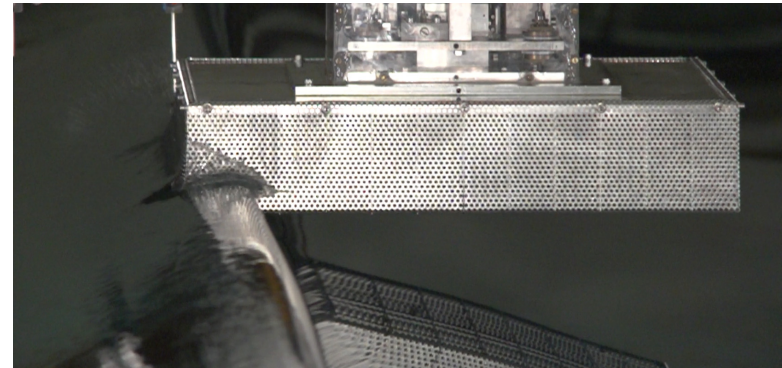

(B)

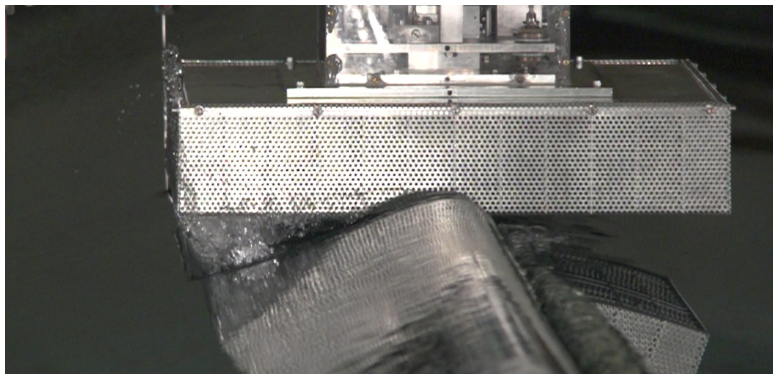

(D)

Figure 16: A sequence of still images describing a WID loading event in an over-turning wave: (A) the wave entering the topside which immediately causes the maximum applied load, $F_{x, \max }$, (B)-(C) the wave crest propagating further into the deck with a large run-up on the front face and (D) the wave profile starting to exit the structure. $\eta$ and $\eta_{c, \text { max }}$. These increas
markedly reduced with $T_{p}$.

In making the observations noted above concerning the relationship between the applied loads and $\Delta \eta$, it should again be noted that the level of inundation is defined by $\Delta \eta=\eta_{c, \max }-h_{d}$. As a result, two approaches can be adopted to obtain the desired $\Delta \eta$ :

(i) Maintain a constant $h_{d}$ and adjust $\eta_{c, \text { max }}$. All of the data presented so far have been based upon this approach.

(ii) Maintain a constant $\eta_{c, \text { max }}$ and adjust $h_{d}$.

Although both approaches allow the required $\Delta \eta$ to be achieved, they are far from identical. For a given $\Delta \eta$, Approach (i) gives a smaller inundation volume $(\Delta \mathcal{V})$, but larger near-crest kinematics when compared to Approach (ii).

The significance of these differences, in terms of the applied loads, requires careful investigation for both non-breaking and breaking wave cases. This is addressed in Figure 18, sub-plots (A), (B) and (C) concerning $T_{p}=1.8 \mathrm{~s}, 1.6 \mathrm{~s}$ and $1.4 \mathrm{~s}$ respectively; the sequence of plots relating to increasing steepness. Considering the non-breaking wave cases (Figure 18(A) and 18(B)), it is clear that Approach (i) and (ii) give very similar results. However, this is not the case if wave breaking occurs (Figure 18(C)). In this case, the data arising from Approach (i) is markedly different. Interestingly, this difference remains significant even when the wave cases involved in the two approaches are both over-turning. This suggests that in breaking waves the near-crest kinematics not only continue to increase as $\eta_{c \text {,max }}$ increases, but that the increase is significantly larger than that arising in non-breaking waves. In terms of the effective rate of momentum transfer, and hence the applied loads, the influence of the increased kinematics appears to outweigh the corresponding changes in the wave shape. This is in marked contrast to the non-breaking wave cases $(\$ 5)$ where the effects of the wave shape dominate. Furthermore, these results offer important insights for the establishment of an effective model testing procedure; Approach (i) being the preferred method. The justification for this is simply that when different levels of inundation occur on a real structure, Approach (i) represents the more realistic scenario. Furthermore, Approach (ii) is non-conservative (Figure 18(C)). Part of the explanation for this is that for the same level of wave inundation, the probability of encountering a breaking wave is substantially reduced in Approach (ii). 


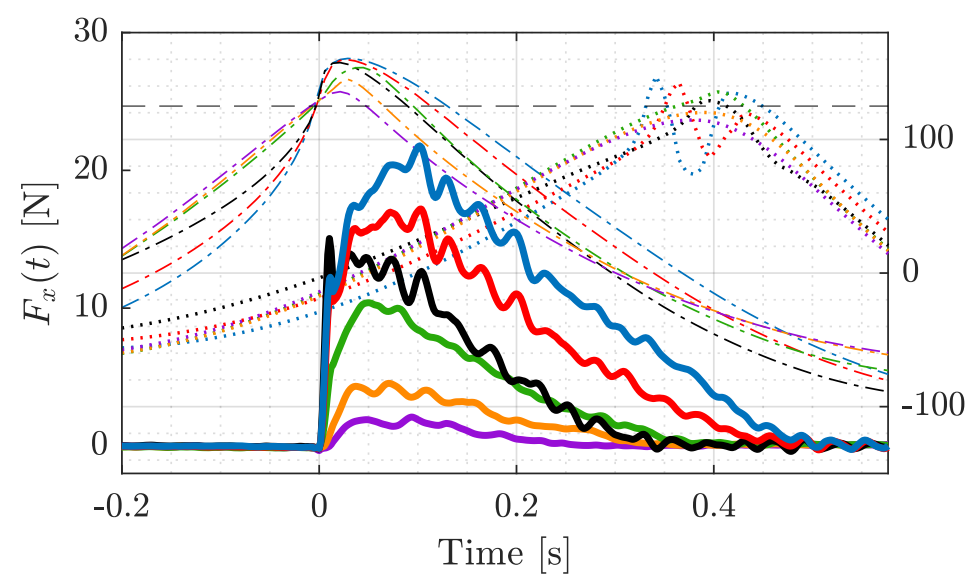

(A)

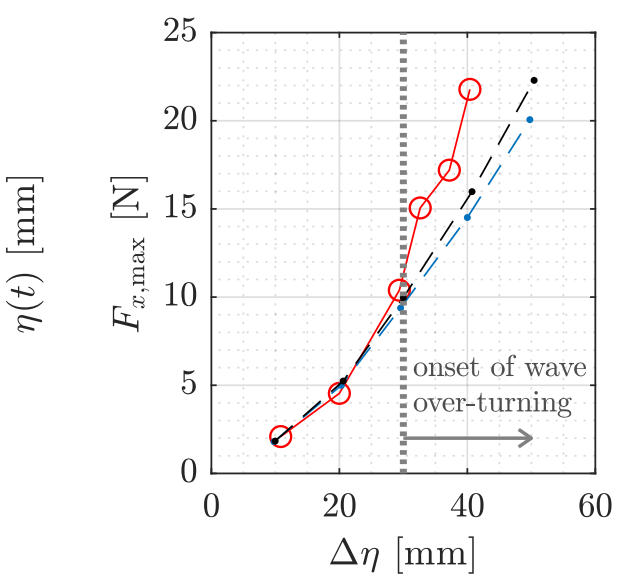

(B)

Figure 17: Horizontal wID loads arising from an increasing $\eta_{c, \text { max }}$ (including breaking waves) while adopting a constant $[--] h_{d}=125 \mathrm{~mm}$. JonswAP spectra, $\gamma=2.5, T_{p}=1.4 \mathrm{~s}$ and $\sigma_{\theta}=0^{\circ}$. (A) [ $[-] F_{x}(t),[-\cdot-] \eta(t)$ at the front and [...] the back faces of the topside. The colours refer to different $\eta_{c, \max }$ : $135 \mathrm{~mm}$ (non-breaking), $145 \mathrm{~mm}$ (limiting), $155 \mathrm{~mm}$ (spilling), $155 \mathrm{~mm}$ (over-turning), $162 \mathrm{~mm}$ (over-turning) and $165 \mathrm{~mm}$ (over-turning). (B) The measured $F_{x, m a x}$ for $[\multimap] T_{p}=1.4 \mathrm{~s}$ with comparisons to $[-\bullet-] T_{p}=1.6 \mathrm{~s}$ and $[-\bullet-] T_{p}=1.8 \mathrm{~s}$.

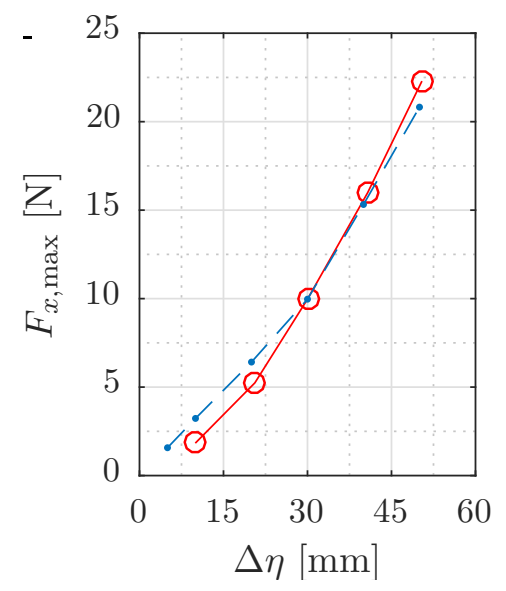

(A) $T_{p}=1.8 \mathrm{~s}$

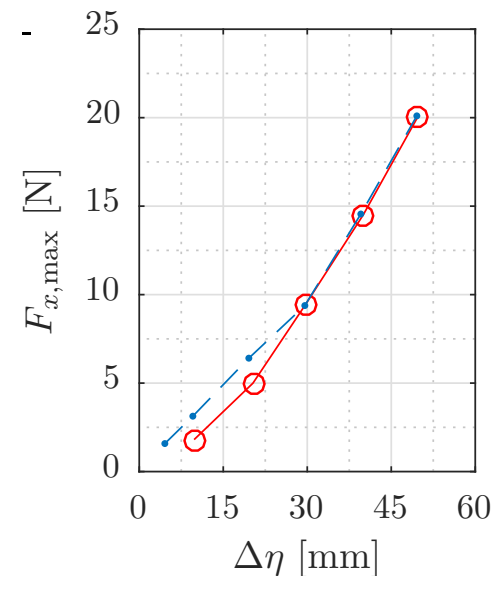

(в) $T_{p}=1.6 \mathrm{~s}$.

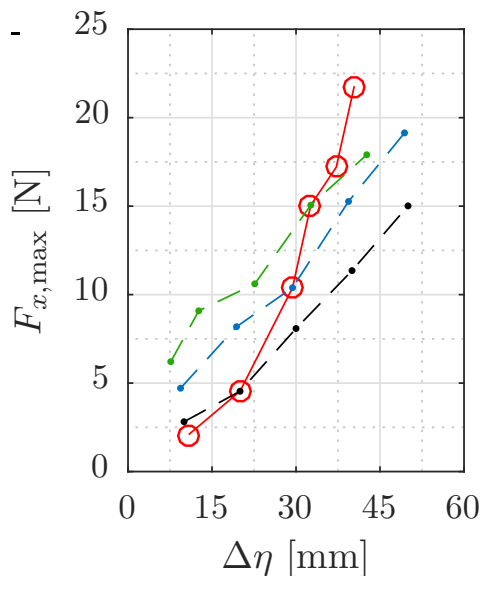

(c) $T_{p}=1.4 \mathrm{~s}$

Figure 18: Measured $F_{x, \max }$, expressed as a function of $\Delta \eta$ for three values of $T_{p}$. All data relate to JonswAP spectra with $\gamma=2.5$ and $\sigma_{\theta}=0^{\circ}$. In each sub-figure, [- - ] represent Approach (i) with a constant $h_{d}=125 \mathrm{~mm}$. For Approach (ii):

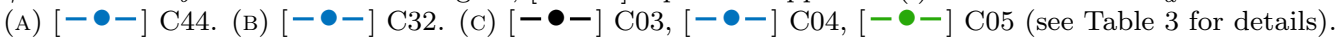

\subsection{Type of wave breaking}

The data provided on Figure 17 and 18 show that as an incident wave breaks, the measured horizontal WID loads show markedly different characteristics. In particular, this difference appears to be most profound for over-turning waves. To investigate this further, Figure 19 provides a direct comparison between $F_{x}(t)$ measured in different types of breaking waves with approximately the same $\Delta \eta$. Whilst $F_{x}(t)$ relating to the limiting and spilling wave cases are closer in form to the previously investigated non-breaking wave cases (Figure 11), $F_{x}(t)$ corresponding to the over-turning wave case features a much larger magnitude and a near-vertical (impulsive) rise in the initial load. The explanation for this again lies in the mass flow rate into the deck and the associated water particle kinematics. Three points merit particular attention:

(1) The near-vertical front face of an over-turning wave gives rise to a large mass flow in a very short time duration. This, in itself, is sufficient to cause an impulsive load. The evidence to support this view has been given in Figure 15. This provides a sequence of still images describing incident waves of varying steepness and, ultimately, different breaker types. It is clear from these images 


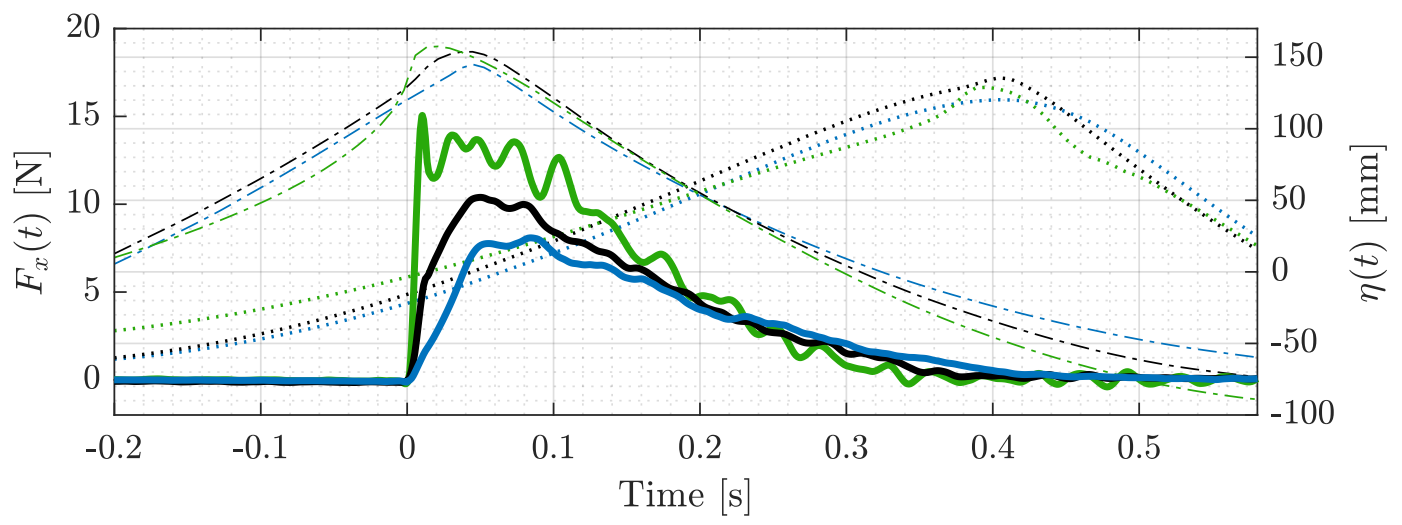

Figure 19: Horizontal WID loads, $F_{x}$, recorded in different types of wave breaking; all data relating to $\Delta \eta=30 \mathrm{~mm}$. $[-] F_{x}(t),[-\cdots-] \eta(t)$ at the front and $[\cdots]$ the back faces of the topside. The colours refer to different wave cases: C03 (limiting), C04 (spilling) and C05 (over-turning).

that the large free surface gradient (upon the first instance of contact with the topside structure) is only relevant to an over-turning wave. In contrast, for the limiting and spilling cases, the topside is progressively submerged, albeit rather quickly.

(2) The near-surface water particle kinematics are substantially larger in an over-turning wave The evidence for this is given in Figure 20. This contrasts the kinematics in a typical non-breaking and over-turning wave with the same crest elevation; the fluid velocities high in the wave crest having been calculated using a fully-nonlinear BEM model. The respective $u_{\max }$ values are given in the figure caption; the $u_{\max }$ associated with the over-turning wave being almost double that of the non-breaking wave.

(3) For a non over-turning incident wave, the fluid momentum is progressively dissipated as the fluid arrives at the topside structure. In contrast, in an over-turning wave the volume of fluid arising at the topside (in a given time interval) is so large that not all the fluid can enter the deck. As a result, some of the fluid will be brought to rest on the boundary of the structure. This provided further blockage to the flow entering the topside. In consequence, a larger volume of fluid is brought to rest (in a very short time interval) leading to a classical impact load. The extent to which this occurs will be dependent upon the porosity or openness of the topside structure. Nevertheless, it is important to note that, depending on the nature of the incident waves, impulsive loading does not solely occur on solid or fully-plated structures.

Taken together, the three points noted above all contribute to a much larger rate of change of momentum at the onset of the loading process. This, in turn, explains the large, impulsive horizontal WID loads observed in the over-turning wave cases. Whilst the present results specifically relate to

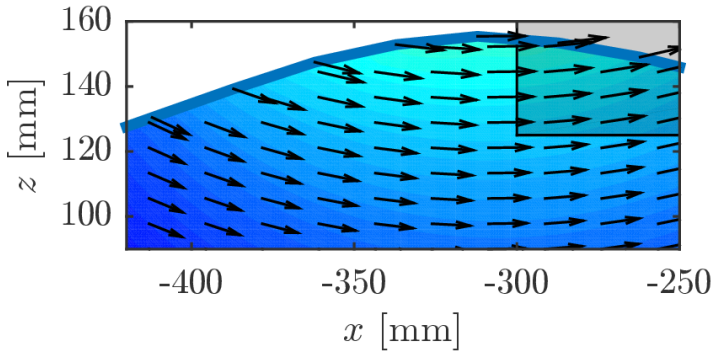

(A)

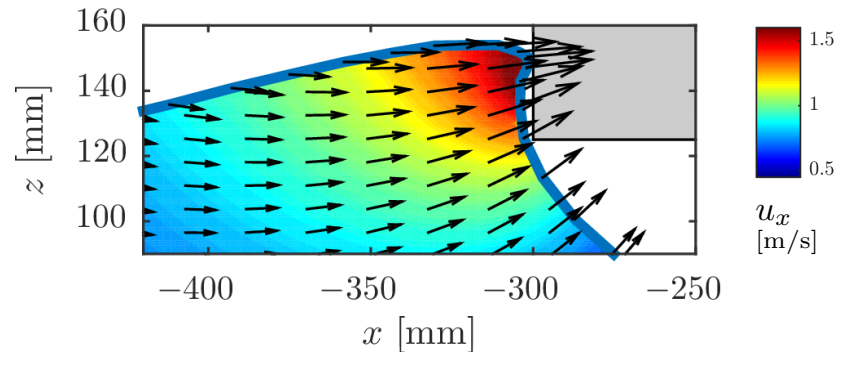

(B)

Figure 20: The water particle kinematics arising high in a wave crest: predictions based on BEM simulations. (A) Wave Case C32 (non-breaking): $u_{\max }=0.92 \mathrm{~m} / \mathrm{s}$ and (B) Wave Case C05 (over-turning): $u_{\max }=1.59 \mathrm{~m} / \mathrm{s}$. In both cases the shaded region represents the topside structure; the coloured contours defining the horizontal water particle velocities and $[\longrightarrow]$ the velocity vectors. 
WID loads, they are consistent with earlier studies (Kjeldsen and Myrhaug, 1979; Chaplin et al., 1992; Stansberg et al., 2012; Stansby et al., 2013) that have demonstrated the importance of wave breaking in 505 respect of the fluid loads acting on either a flat plate or a fixed vertical column. However, when compared to the WID loads reported by Stansberg et al. (2004), the present records exhibit a more gradual reduction in the applied load following the maximum. This is a direct consequence of the difference in the porosity of the topside structure; the earlier work by Stansberg et al. (2004) involving a fully-plated structure. A detailed investigation of the role of topside porosity is presently on-going.

\subsection{Variations with other wave parameters}

The observations outlined above, concerning the importance of wave breaking and the fundamental changes it makes to the relative importance of the wave shape and the water particle kinematics, are consistently observed across a broad range of wave parameters. Evidence of this is presented as follows:

Figure 21 presents the variation in the horizontal wID load, $F_{x}(t)$, with the spectral peak period, $T_{p}$; all other parameters held constant. When compared to Figure 12, the wave case associated with the smallest $T_{p}$ is now over-turning. As a result, it now gives the largest load (Figure 21) rather than the smallest (Figure 12). Moreover, with the deck elevation held constant at $h_{d}=125 \mathrm{~mm}$, an increase in the crest elevation from $145 \mathrm{~mm}$ (on Figure 12) to $155 \mathrm{~mm}$ (on Figure 21) produces a $50 \%$ increase in the deck inundation, $\Delta \eta$. As a result, the non-breaking wid loads (corresponding to $T_{p}=1.6 \mathrm{~s}$ and $1.8 \mathrm{~s}$ ) increase by almost $100 \%$. This is consistent with the data presented in Figure 11(B). However, if as a consequence of the increased elevation the wave now over-turns, the maximum loads increase by more than $350 \%$. This is a direct consequence of the increased fluid velocities and the fact that the loads are proportional to the square of these velocities. This occurs despite a reduction in the size of the incident wave crest due to an increase in the steepness. In considering this largest breaking wave load, corresponding to $T_{p}=1.4 \mathrm{~s}$ on Figure 21, it is also important to note that this is not a classical impact load, involving a large force for a very short duration. Indeed, whilst the loading time-history, $F_{x}(t)$, is markedly different, involving a very rapid initial rise, the total duration of the load is comparable to the non-breaking wave cases.

Figure 22 concerns the variation in $F_{x}(t)$ with the directional spread, $\sigma_{\theta}$, for a constant $T_{p}=1.4 \mathrm{~s}$. $\sigma_{\theta}=10^{\circ}$ are over-turning, the degree of wave breaking progressively reduces as $\sigma_{\theta}$ increases due to a reduction in the wave steepness. Once again, the overturning waves produce a distinct, impulsive, $F_{x}(t)$, with a very rapid rise in the initial load. Moreover, when compared to Figure 13, the reduction in $F_{x, \max }$ with $\sigma_{\theta}$ is more rapid for $0^{\circ} \leqslant \sigma_{\theta} \leqslant 15^{\circ}$. This is primarily the result of the reduced water particle kinematics high in the wave crest as the waves cease 535 to be over-turning.

Figure 23 concerns the variation in $F_{x}(t)$ with the spectral bandwidth; the latter defined by the peak enhancement factor, $\gamma$, and includes a comparative regular wave case representing the limit of narrow-bandedness. All cases correspond to a reduced spectral peak period of $T_{p}=1.4 \mathrm{~s}$, with all other parameters held constant. As such, the wave cases are closely related to those addressed in Figure 14(A). ${ }_{540}$ However, in contrast to the earlier results, the reduced $T_{p}$ ensures that the wave case relating to the largest spectral bandwidth $(\gamma=1)$ is now an over-turning wave event. As a result, this now defines the largest loading event rather than the smallest. Importantly, the regular wave is no longer the most conservative, despite being associated with the largest volume of water within the incident wave crest.

Taken together, the data presented in Figures 17-23 confirm that the nature of any WID loads continues to be dependent upon both the wave shape and near-surface water particle kinematics. However, the increase in the latter due to the occurrence of wave breaking, particularly wave over-turning, ensures that the kinematics exert a controlling influence over both the magnitude and the time-history of the applied WID loads. This is in stark contrast to observations outlined in $\S 5$ relating to non-breaking waves.

\section{Concluding remarks}

The present paper has documented an experimental study of WID loading, involving a generic topside structure, subject to a wide range of deterministic focused wave events; the latter being representative of the largest waves arising in a random sea. The purpose of these tests has been to provide a fundamental understanding of the applied loads. Specifically, to investigate the relative importance of the various incident wave properties, including the crest elevation, spectral peak period, directional spread, spectral 


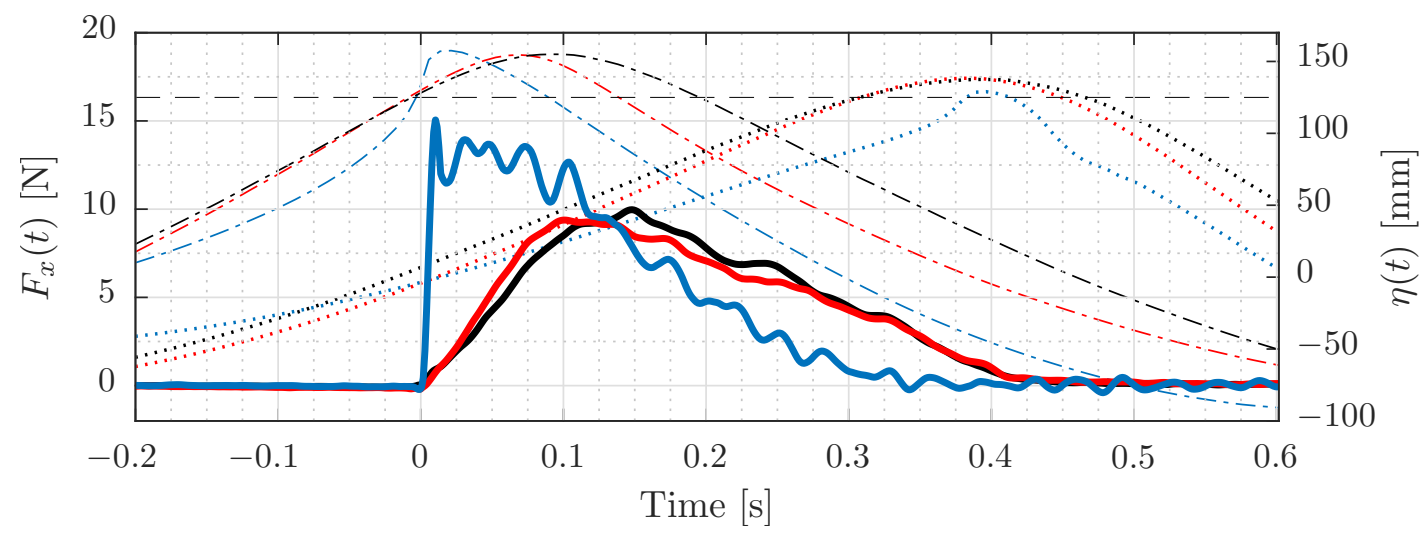

Figure 21: The variation in the horizontal wID loads for different $T_{p}$ (including breaking waves), with a constant $\Delta \eta$. $[--] h_{d}=125 \mathrm{~mm} . \eta_{c, \max }=155 \mathrm{~mm}$, JONSwAP spectra, $\gamma=2.5$, uni-directional. $[-] F_{x}(t),[-\cdot-] \eta(t)$ at the front and $[\cdots]$ ] the back faces of the topside. The colours refer to different $T_{p}: 1.4 \mathrm{~s}$ (C05, over-turning), $1.6 \mathrm{~s}(\mathrm{C} 32)$ and $1.8 \mathrm{~s}(\mathrm{C} 44)$.

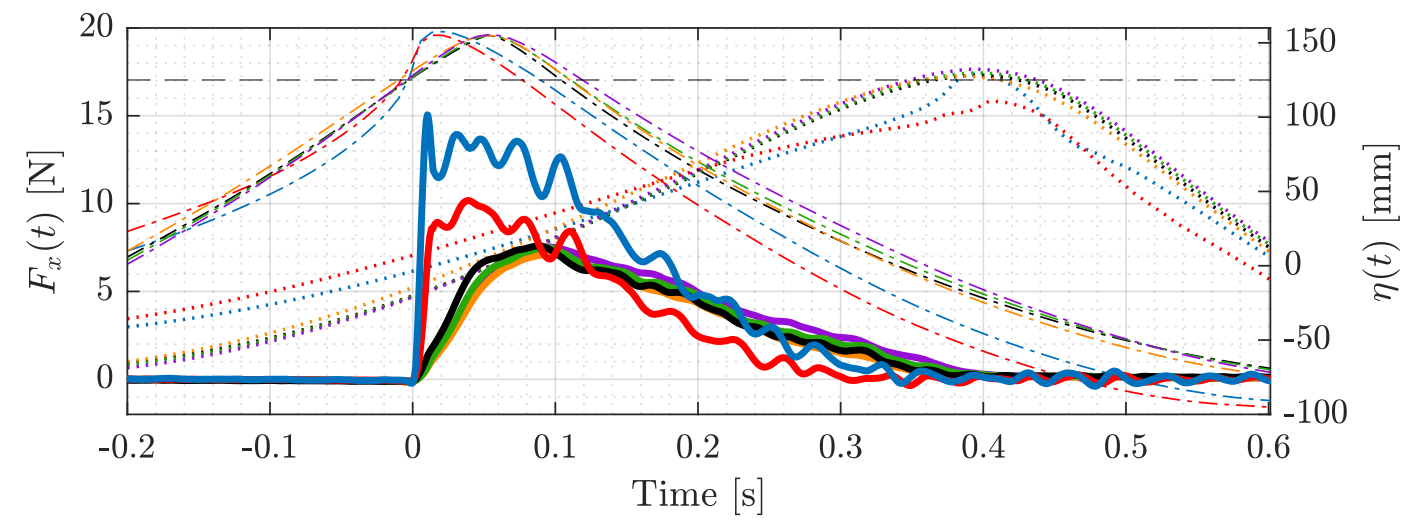

Figure 22: The variation in the horizontal wID loads due to increasing levels of directional spread, $\sigma_{\theta}$ (including breaking waves), for a constant $\Delta \eta \cdot \eta_{c, \max }=155 \mathrm{~mm}$, JONSWAP spectra, $\gamma=2.5, T_{p}=1.4 \mathrm{~s}$. [ - ] $h_{d}=125 \mathrm{~mm},\left[{ }^{-}\right] F_{x}(t)$,

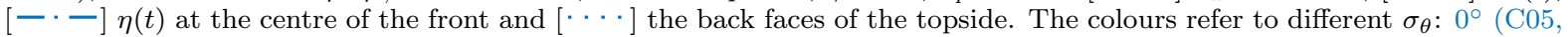
over-turning), $10^{\circ}$ (over-turning), $15^{\circ}$ (spilling), $20^{\circ}$ (spilling), $25^{\circ}$ (limiting) and $30^{\circ}$ (non-breaking).

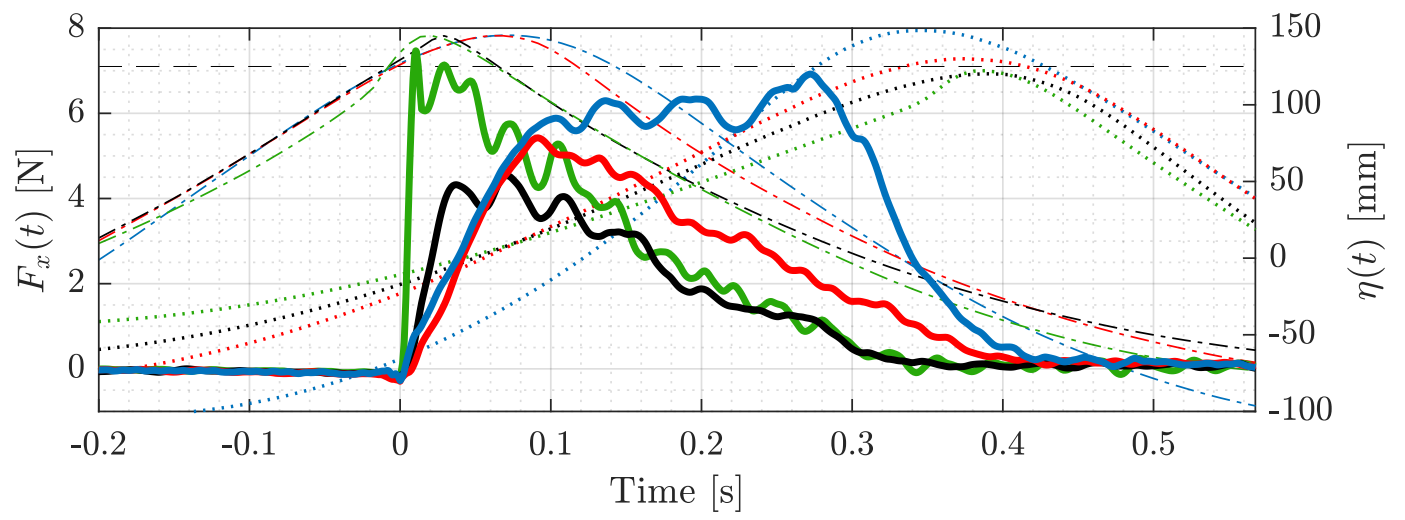

Figure 23: The variation in the horizontal WID loads due to different spectral bandwidths (including breaking waves), for a constant $\Delta \eta \cdot \eta_{c, \max }=145 \mathrm{~mm}$, JONSWAP spectra, $T_{p}=1.4 \mathrm{~s}$, uni-directional. (A) $[--] h_{d}=125 \mathrm{~mm},\left[\frac{}{-}\right] F_{x}(t)$, $[-\cdot-] \eta(t)$ the front and $[\cdots]$ the back faces of the topside. The colours refer to different spectral bandwidth: regular wave, $\gamma=5, \gamma=2.5$ (C03, limiting) and $\gamma=1$ (over-turning). 
bandwidth and, most importantly, the occurrence of varying types of wave breaking. Having considered many aspects of the incident wave field, the applied wID loading has been shown to be primarily dependent upon the combined effects of: (a) the shape of the wave crest and (b) the water particle kinematics generated therein. This is entirely consistent with the origins of the applied force being equal to the rate of transfer of momentum from the wave crest into the topside structure. Whilst the wave shape and the water particle kinematics are clearly related, the relative importance of the two effects are such that in non-breaking waves, changes in the crest shape exert a controlling influence. In contrast, with the occurrence of wave breaking, particularly wave over-turning, the increase in the fluid velocities high in the wave crest is such that the kinematics become dominant.

This has important practical implications, not least because the occurrence of large over-turning waves in intermediate and deep water depths are seldom addressed as part of the design or re-assessment of fixed offshore structures, particularly in respect of wave-in-deck loading. Indeed, it is curious to note that whilst the importance of wave breaking is tentatively acknowledged in respect of horizontal slamming loads (DNV-GL, 2019a) acting on large diameter columns or large plated bodies (including the front face of a semi-submersible floating structure), this has not yet been extended to the calculation of wave-in-deck loads on fixed structures. Specifically, the present international standards either allow (and in some instances recommend) the use of an 'equivalent' regular wave as the basis for wave-in-deck load predictions. Since these will neither model the crest shape, $\eta(x, y, t)$, nor the associated water particle kinematics, $\mathbf{u}(x, y, z, t)$, the resulting load predictions are unlikely to be accurate without extensive and problem-specific calibration. Moreover, since large breaking waves occurring in irregular or random seas will be very different to the prediction of a regular wave model based upon a local wave period and wave height, the largest wave-in-deck loading events, and hence those most relevant to design/re-assessment, are likely to be the least well-predicted. Most importantly, the results presented herein suggest that present practice is likely to yield non-conservative load predictions, unless they are based upon detailed physical model tests involving long random wave simulations.

\section{References}

API, 2014. Structural Integrity Management of Fixed Offshore Structures API RP 2SIM. American Petroleum Institute (API), Washington D.C.

Archibald, S., 2011. Modelling of Extreme Ocean Waves Using High Performance Computing. Ph.D. thesis. Imperial College London. South Kensington, London SW7 2AZ.

Boccotti, P., 1983. Some new results on statistical properties of wind waves. Applied Ocean Research 5, $134-140$.

Chaplin, J., Greated, C., Flintham, T., Skyner, D., 1992. Breaking wave forces on a vertical cylinder. Technical Report. Department for En- 306 ergy within the SERC/MTD managed programme, Ocean Engineering Research Centre, City University. London.

590 Christou, M., 2009. Fully Nonlinear Computations of Waves and Wave-structure Interaction. Ph.D. thesis. Imperial College London. South Kensington, London SW7 2AZ.

Christou, M., Ewans, K., 2014. Field measurements of rogue water waves. Journal of Physical Oceanography 44, 2317-2335. URL: http://dx.doi.org/10.1175/JPO-D-13-0199.1, doi:10.1175/ JPO-D-13-0199.1.

Clough, R., Penzien, J., 2003. Dynamics of Structures. $3^{\text {rd }}$ ed., Computers \& Structures.

DNV-GL, 2019a. Offshore Technical Guidance DNVGL-OTG-14: Horizontal wave impact loads for column stabilised units. DNV-GL.

DNV-GL, 2019b. Recommended Practice DNVGL-RP-C205: Environmental Conditions and Environmental Loads. DNV-GL, Norway.

Ewans, K.C., 1998. Observations of the directional spectrum of fetch-limited waves. Journal of Physical Oceanography 28, 495-512. doi:10.1175/1520-0485 (1998) 028<0495: OOTDSO>2.0. C0; 2. 
Graaf, J.W.v.d., Tromans, P., Vanderschuren, L., 1995. Wave loads on decks. Shell Offshore Structures Engineering Newsletter .

Hague, C.H., Swan, C., 2009. A multiple flux boundary element method applied to the description of surface water waves. Journal of Computational Physics 228, 5111-5128.

Haley, J.F., 2016. Fluid forcing in the crests of large ocean waves. Ph.D. thesis. Imperial College London. South Kensington, London SW7 2AZ.

Haley, J.F., Swan, C., Gibson, R., 2014. An experimental investigation of wave impact loads on a slender horizontal cylinder, in: ASME 2014 33rd International Conference on Ocean, Offshore and Arctic Engineering, American Society of Mechanical Engineers.

Hall, T., 2012. Hurricane isaac costs Gulf of Mexico $\$ 500 \mathrm{~m}$ to $\$ 1 \mathrm{bn}$ (url: http://www.offshoretechnology.com/contractors/maintenance/welaptega-marine/presshurricane-isaac-costs-gulf-ofmexico-500m-to-1bn.html) [accessed: 2015-07-02].

Hasselmann, K., Barnett, T., Bouws, E., Carlson, H., Cartwright, D., Enke, K., Ewing, J., Gienapp, H., Hasselmann, D., Kruseman, P., et al., 1973. Measurements of wind-wave growth and swell decay during the JOint North Sea WAve Project (JONSWAP). Technical Report. Deutches Hydrographisches Institut.

ISO, 2013. Petroleum and natural gas industries - Fixed steel offshore structures (ISO 19902:2007/Amd.1:2013). International Organization for Standardization (ISO).

Kaiser, J.B., Chambers, M.D., 2017. Chapter 13: Offshore platforms and mariculture in the US. Aquaculture perspective of multi-use sites in the open ocean: The untapped potential for marine resources in the anthropocene (url: https://www.researchgate.net/figure/offshore-platform-damaged-during-ahurricane-off-of-louisiana_fig2_315874734) [accessed: 2019-02-20]. Springer Open Access (Creative Commons Lincence CC BY 4.0).

Kaplan, P., Murray, J.J., Yu, W.C., 1995. Theoretical analysis of wave impact forces on platform deck structures, in: OMAE 1995, 14th International Conference on Offshore Mechanics \& Arctic Engineering, ASME, Copenhagen, Denmark. p. 189.

Kjeldsen, S.S., Myrhaug, D.S., 1979. Breaking waves in deep water and resulting wave forces, in: Offshore Technology Conference, Offshore Technology Conference.

Latheef, M., 2014. Surface wave statistics in directionally spread seas. Ph.D. thesis. Imperial College London. South Kensington, London SW7 2AZ.

Latheef, M., Swan, C., 2013. A laboratory study of wave crest statistics and the role of directional spreading. Proceedings of the Royal Society of London A: Mathematical, Physical and Engineering Sciences 469.

Lindgren, G., 1970. Some properties of a normal process near a local maximum. The Annals of Mathematical Statistics, $1870-1883$.

Ma, L., 2017. The effective modelling of wave-in-deck loads. Ph.D. thesis. Imperial College London. South Kensington, London SW7 2AZ.

Masterton, S.R., Swan, C., 2008. On the accurate and efficient calibration of a 3D wave basin. Ocean ${ }_{640} \quad$ Engineering 35, 763-773.

NORSOK, 2017. N-003 Actions and action effects. 3 ed., Norwegian Technology Standards Institution (NORSOK), Mustads vei 1, NO-0283 Oslo, Norway.

Paganie, D., 2008. Ike topples old platforms (url: https://www.offshore-mag.com/articles/print/volume68/issue-10/departments/gulf-of-mexico/gulf-of-mexico.html). [accessed: 2019-02-20].

Spinneken, J., Swan, C., 2009a. Second-order wave maker theory using force-feedback control. part i: A new theory for regular wave generation. Ocean Engineering 36, 539-548. 
Spinneken, J., Swan, C., 2009b. Second-order wave maker theory using force-feedback control. part ii: An experimental verification of regular wave generation. Ocean engineering 36, 549-555.

Spinneken, J., Swan, C., 2012. The operation of a 3D wave basin in force control. Ocean Engineering $55,88-100$.

Stansberg, C.T., Baarholm, R., Fokk, T., Gudmestad, O.T., Haver, S., 2004. Wave amplification and possible deck impact on gravity based structure in 10-4 probability extreme crest heights, in: ASME 2004 23rd International Conference on Offshore Mechanics and Arctic Engineering, American Society of Mechanical Engineers. pp. 911-920.

Stansberg, C.T., Berget, K., Graczyk, M., Muthanna, C., Pakozdi, C., 2012. Breaking wave kinematics and resulting slamming pressures on a vertical column, in: ASME 2012 31st International Conference on Ocean, Offshore and Arctic Engineering, American Society of Mechanical Engineers. pp. 679-689.

Stansby, P.K., Devaney, L.C., Stallard, T.J., 2013. Breaking wave loads on monopiles for offshore wind turbines and estimation of extreme overturning moment. IET Renewable Power Generation 7, 514-520.

Tromans, P.S., Anaturk, A.R., Hagemeijer, P., 1991. A new model for the kinematics of large ocean waves-application as a design wave, International Society of Offshore and Polar Engineers.

Tychsen, J., Risvig, S., Hansen, H.F., Hansen, N.E.O., Stevanato, F., 2016. Summary of the impact on structural reliability of the findings of the tyra field extreme wave study 2013-15, in: 3rd Offshore Structural Reliability Conference, pp. 14-16.

U.S. Department of State, 2006. Hurricane katrina: What government is doing (url: http://www.state.gov/documents/organization/150082.pdf) [accessed: 2015-07-02].

Young, I.R., Zieger, S., Babanin, A.V., 2011. Global trends in wind speed and wave height. Science 332, $451-455$.

\section{Declare of interest}

None. 